\title{
Implementation of the classical plasma-fluid model for simulation of dielectric barrier discharge (DBD) actuators in OpenFOAM
}

\author{
M. Abdollahzadeh*, J.C. Pascoa, P.J. Oliveira
}

Departamento de Engenharia Electromecanica, C-MAST - Center for Mechanical and Aerospace Sciences and Technologies, Universidade da Beira Interior, Covilha, Portugal

\section{A R T I C L E I N F O}

\section{Article history:}

Received 11 March 2015

Revised 7 December 2015

Accepted 20 January 2016

Available online 30 January 2016

\section{Keywords:}

DBD plasma actuator

Plasma-Fluid model

Super-time-stepping

Electric discharge

OpenFOAM

\begin{abstract}
A B S T R A C T
To simulate the coupled plasma and fluid flow physics of dielectric-barrier discharge, a plasma-fluid model is utilized in conjunction with a compressible flow solver. The flow solver is responsible for determining the bulk flow kinetics of dominant neutral background species including mole fractions, gas temperature, pressure and velocity. The plasma solver determines the kinetics and energetics of the plasma species and accounts for finite rate chemistry. In order to achieve maximum reliability and best performance, we have utilized state-of-the-art numerical and theoretical approaches for the simulation of DBD plasma actuators. In this respect, to obtain a stable and accurate solution method, we tested and compared different existing numerical procedures, including operator-splitting algorithm, super-timestepping, and solution of the Poisson and transport equations in a semi-implicit manner. The implementation of the model is conducted in OpenFOAM. Four numerical test cases are considered in order to validate the solvers and to investigate the drawbacks/benefits of the solution approaches. The test problems include single DBD actuator driven by positive, negative and sinusoidal voltage waveforms, similar to the ones that could be found in literature. The accuracy of the results strongly depends to the choice of time step, grid size and discretization scheme. The results indicate that the super-time-stepping treatment improves the computational efficiency in comparison to explicit schemes. However, the semiimplicit treatment of the Poisson and transport equations showed better performance compared to the other tested approaches.
\end{abstract}

(C) 2016 Elsevier Ltd. All rights reserved.

\section{Introduction}

Active flow control techniques that use dielectric barrier discharge (DBD) are considered very promising. DBD plasma actuators have shown a great potential for flow control purposes, especially regarding aeronautical applications. In general, DBD plasma actuator can induce a body force on a gas and heat up that gas. Body forces are exerted on charged species (electrons and ions) by an electric field and become coupled to the bulk gas motion by particle collisions. These forces can then be used to control the flow.

The optimization of DBD plasma actuator performance and further improvement in plasma flow control technology rely on comprehensive numerical modeling and robust computational tools. There have been a number of numerical studies on DBD plasma actuators. Computational models of plasma discharges may be divided into four types, namely: i) fluid models [1-4]; ii) kinetic/particle models [5]; iii) hybrid approaches [6]; and iv)

\footnotetext{
* Corresponding author. Tel.: +351 925467631; fax: +351 275329972.

E-mail addresses: mm.abdollahzadeh@yahoo.com (M. Abdollahzadeh), pascoa@ubi.pt (J.C. Pascoa), pjpo@ubi.pt (P.J. Oliveira).
}

simple phenomenological or empirical models [7,8]. A detailed review of different approaches for modeling plasma actuators can be found in [9-11]. Jayaraman and Shyy [10] and Jayaraman [12] also reviewed the first-principle based hydrodynamic plasma model. Among these models, plasma fluid models are computationally less expensive and are suitable for spatially resolving the various physical phenomena occurring in actual plasma. Plasma-fluid models treat the plasma as a fluid of freely moving charged particles and end-up in a set of drift-diffusion type transport equations. The chemical species present in the plasma, such as electrons and ions, are tracked using transport equations and the essential plasma physics, such as ionization, recombination and streamer propagation, are all modeled. In general, these models are capable of accurately resolving and predicting plasma phenomena. However, the solution of these equations requires very small spatial resolution. Such limitation also imposes a significant restriction on the numerical time step and prohibits the computation of high voltages at $\mathrm{kHz}$ frequencies.

In the first modeling attempts of DBD plasma actuators using plasma fluid models, which were accomplished by Roy [13] and Singh and Roy [14], continuity equations for the electron and 


\section{Nomenclature}

$D_{k} \quad$ charged particle diffusion coefficient $\left(\mathrm{m}^{2} \mathrm{~s}^{-1}\right)$

$e \quad$ electron elementary charge $e=1.6022 \times 10^{-19} \mathrm{C}$

$\vec{E} \quad$ electric field vector $\left(\mathrm{kg} \mathrm{m} \mathrm{s}^{-3} \mathrm{~A}^{-1}\right)$

$f(t) \quad$ voltage waveform

$\tilde{\mathrm{f}}$ force density in electrohydrodynamics (EHD) $\left(\mathrm{N} \mathrm{m}^{-3}\right)$

$\mathrm{H}$ homogenous operator

$\vec{j} \quad$ total electric current flux $\left(\mathrm{A} \mathrm{m}^{-2}\right)$

$k_{B} \quad$ Boltzmann constant $\left(\mathrm{m}^{2} \mathrm{~kg} \mathrm{~s}^{-2} \mathrm{~K}^{-1}\right)$

Kn Knudsen number

$L \quad$ loss term $\left(\mathrm{m}^{-3} \mathrm{~S}^{-1}\right.$

$m \quad$ mass of particle $(\mathrm{kg})$

$n_{0} \quad$ initial charge particle density $\left(\mathrm{m}^{-3}\right)$

$n_{k} \quad$ number charge particle density $\left(\mathrm{m}^{-3}\right)$

$\vec{n} \quad$ unit normal vector

$\mathrm{P} \quad$ parabolic operator

$p \quad$ pressure $\left(\mathrm{N} \mathrm{m}^{-2}\right)$

$r \quad$ recombination coefficient $\left(\mathrm{m}^{3} \mathrm{~s}^{-1}\right)$

$\mathrm{R} \quad$ reaction operator

$S \quad$ source term $\left(\mathrm{m}^{-3} \mathrm{~s}^{-1)}\right.$

$t \quad$ time (s)

$T \quad$ temperature $(\mathrm{K})$

$\Delta t \quad$ time step

$\vec{v}_{k} \quad$ species velocity $\left(\mathrm{m} \mathrm{s}^{-1}\right)$

$\vec{v}_{b} \quad$ background flow velocity $\left(\mathrm{m} \mathrm{s}^{-1}\right)$

$V_{0} \quad$ voltage amplitude (V)

Greek symbols

$\lambda$ mean free path $(\mathrm{m})$

$\vec{\Gamma}_{k} \quad$ fluxes of charged particles $\left(\mathrm{m}^{-2} \mathrm{~s}^{-1}\right)$

$\varepsilon_{0} \quad$ permittivity of space $\left(\mathrm{Fm}^{-1}\right)$

$\varphi \quad$ electric potential $(\mathrm{V})$

$\sigma \quad$ surface charge density $\left(\mathrm{C} \mathrm{m}^{-2}\right)$

$\mu_{k} \quad$ charged particle mobility $\left(\mathrm{m}^{2} \mathrm{~V}^{-1} \mathrm{~s}^{-1}\right)$

$\alpha \quad$ ionization coefficient, $\left(\mathrm{m}^{-1}\right)$

$\eta \quad$ attachment coefficient $\left(\mathrm{m}^{-1}\right)$

$\gamma \quad$ secondary electron emission

$\Delta \quad$ characteristic dimension of boundary cell (normal to the boundary) (m)

\section{Subscripts}

$d \quad$ dielectric

e electrons

ep electron-ion

$k \quad$ particle type

$p \quad$ positive ion

$n \quad$ negative ion

np ion-ion

0 reference state

$s \quad$ surface

sec secondary

th thermal

air air side

\section{Superscripts}

Int adjacent internal grid cell center

ion densities were the only equations considered, solved as Poisson type equations. However, the processes of surface charge accumulation, recombination and secondary emission, which are essential for the plasma discharges, were not taken into account. Later, improved physical-based modeling procedures were developed, based on better understanding of the fundamentals of the essential physics of DBD actuators. Duan et al. [15] studied numerically the uniformity of glow discharge of DBD actuators in subatmospheric conditions. Nishida and Abe [16], Boeuf et al. [17], and Unfer and Boeuf [18] analyzed the dependency of the discharge mode on the driving voltage waveform, ranging from a simplified case with a constant voltage pulse to time-varying voltage of various shapes, such as a ramp, saw tooth (triangular applied voltage waveform), sinusoidal and nanosecond applied voltage waveform. Contribution of negative and positive ions on force generation in DBD plasma actuators was also studied by Lagmich et al. [19].

More recently, the attempts to the modeling of DBD plasma actuators were aimed at achieving a complete and comprehensive physically-based model, incorporating all essential physics of DBD plasma actuators and using modern numerical methods for efficient simulation. Along this line, Roy and Gaitonde [20] coupled a plasma model with an hydrodynamic model for the description of the induced gas flow. Singh and Roy [1], considered the effects of Joule heating in the plasma, dielectric heating, and electro-dynamic force. Boeuf and Pitchford [21], computed the force per unit volume acting on the flow, due to the effect of the plasma actuator, based on a two-dimensional fluid model of the surface discharge and by considering positive ions and electrons with the nitrogen as the background inert gas. They have assumed that the gas flow velocity is small with respect to the charged particle drift velocities, and that the gas flow does not significantly affect the plasma (the so-called one-way coupling). Recently, Shang and Huang $[22,23]$ presented a physical based modeling procedure of DBD actuators by adopting the drift-diffusion as an approximation to the species transport in the non-equilibrium air plasma and by considering the process of ionization, electron attachment, detachment, bulk, and ion-ion recombination. Moreover, the effects and quantifications of Joule heating, periodic electrostatic force, as well as the Lorentz acceleration for flow control were also considered. Singh and Roy [24] extended the asymmetric DBD model by considering real gas air chemistry using a selfconsistent multibody system of plasma, dielectric and neutral gas. Likhanskii et al. [25] developed a detailed physical model for asymmetric DBD in air considering both positive and negative ions, and also electrons with relevant plasma kinetics such as recombination, ionization, attachment and secondary electron emission from metallic and dielectric surfaces. They used a second-order accurate MacCormack scheme, with flux corrected transport, as the algorithm for the numerical simulation of the plasma kinetics. The computed force and heating rate from the plasma model was then coupled to a viscous flow solver. Recently Unfer et al. [26] developed an asynchronous scheme with local time stepping for solving a 2D discharge model. This technique reduces the CPU time required for the explicit solution of the Poisson and transport equations while permitting the use of high order spatial schemes. Wang and Roy [27] used a three-species physical model for DBD actuators. The plasma model was loosely coupled with the compressible Navier-Stokes equations through momentum and energy source terms. The influence of DBD actuator was considered through a time averaged electrohydrodynamic (EHD) force and power deposition to the neutral gas. The power deposition accounts for ion Joule heating and a certain percentage of electron Joule heating.

This brief review provides details of some of the numerical techniques that have been used in plasma-fluid modeling, however, it should be added that there are no published reports, as far as we are aware, in which a comparison between those approaches is carried out and their merits discussed. In this paper the coupled plasma-fluid flow physics simulations were conducted after implementation of a suitable physical model, represented by a set of partial differential equations, in a computational fluid dynamics code. As a test platform, we choose the unstructured, finite volumebased method in the open-source $\mathrm{C}^{++}$library OpenFOAM. The 
plasma fluid was then implemented in the OpenFOAM CFD toolbox [28]. Formation of plasma discharges involves multiple-scale processes such as convection, diffusion and reaction/ionization mechanisms which make the transport equations of the plasma dynamics stiff. To obtain a stable and accurate solution, several numerical procedures, including a sequential finite-volume operator-splitting algorithm [29,30], semi-implicit treatment of Poisson equation and super-time-stepping, were implemented and tested. The application of super-time-stepping approach in modeling DBD plasma actuators is reported here for the first time. In addition, the influence of the selected time step and grid size on the accuracy of the results obtained by each numerical scheme is discussed. Then, a body force or localized heating treatment is devised to link the plasma dynamics and fluid dynamics. The fluid dynamics is solved using a density-based algorithm in a multi-region framework. The rest of the paper is arranged in the following format: a detailed description of the fluid-plasma model is provided in Section 2 and the numerical model is summarized in Section 3. Section 4 presents the results. Finally, conclusions are drawn in Section 5 .

\section{Governing equations and boundary conditions}

DBD can be operated at sub-atmospheric, atmospheric and higher than atmospheric pressures. However, atmospheric-pressure plasmas have higher technical importance in contrast with lowpressure plasma or high-pressure plasma. Production of low or high pressure plasma needs cost-intensive chambers making them highly expensive. For the type of applications, we envisage, where DBDs are used for active flow separation control and to improve the aerodynamics of high-altitude aircraft [7,8,31-33], the plasma will be subject to close to atmospheric pressures. By assuming that DBD is operating at atmospheric pressure, a reasonable and computationally efficient approximation can be made using a timedependent plasma-fluid model instead of a kinetic model. Plasma fluid models (hydrodynamic models) are based on the Boltzmann equation and its moments [34-36]. The fluid model description using the continuity equations for the various particle densities is satisfactory at atmospheric pressure, when the particles relaxation times for equilibrium of momentum and energy are generally small compared with any macroscopic time scale variation of the system [11]. The core of the model consists of solving the compressible Navier-Stokes equation, the Maxwell equation for the electromagnetic field and multiple species continuity equations, including non-equilibrium chemical reactions, to obtain the spatial-temporal distributions of each particle species and electric field.

For simplicity, the plasma is considered as a multi-component fluid considering global categories of charged particles, comprising the four primary species, namely, neutrals, positive and negative ions and electrons. Multiple charged ions will be neglected. This implies the assumption that positive ions or negative ions are in close equilibrium with other positive or negative ions which results in one type of effective positive ion and one type of effective negative ion [37]. The model does not describe the kinetics of excited species and their influence on the plasma evolution. The model only considers that at any instance during the discharge, the ionized species concentration is the net balance between ionization, detachment, attachment and recombination processes. Moreover, the model accounts for the charging of the dielectric surface due to the incident charged particles.

In modeling gaseous plasma discharges using a fluid model, several important assumptions have to be made. Here, it is assumed that the DBD plasma is a weakly ionized plasma which is operating at atmospheric conditions. The imbalance of net space charge due to the charged species densities will generate self- consistent electric forces. In the absence of externally magnetic field and by assuming that the current densities are small (weakly ionized plasma), self-consistent magnetic fields can be safely neglected and the Maxwell equations can be reduced to solving a single Poisson equation for the electric potential [23]. We assumed two characteristic temperatures, the heavy species temperature and a separate electron temperature [38]. Each temperature could be obtained separately by solving energy conservation equations. However, as ions tend to thermalize effectively with neutrals by frequent charge exchange collisions, it might be assumed that the ion temperature is in the order of the neutral gas temperature (It is assumed that all heavy species are in thermal equilibrium with each other). In other words, since the ion mass is very nearly the same as the mass of the background gas, the ions are assumed to be in thermal equilibrium with the background, and no energy equation is solved for the ions. For the electrons, due to their lower mass, the exchange of kinetic energy with the other particles is poor, and their temperature can be significantly higher than the other species. Thus, for simplification, a high constant electron temperature is assumed [39].

The species flux $\vec{\Gamma}=n \vec{v}_{s}$ can be obtained by solving separate momentum equations for each species. However, at atmospheric pressure, a reasonable and computationally efficient approximation can be made using the so called drift-diffusion approximation [40]. In this approximation, the fluxes of charged particles are calculated by neglecting the inertial and unsteady terms of the momentum equation and balancing the thermodynamic pressure gradient with the drift force and collision terms. This assumption for reduction of the momentum equation for the species is valid at high pressures (e.g. atmospheric conditions) [39]. Such an approximation requires the pressure tensor to be isotropic and is strictly valid only for a Maxwellian distribution. Moreover, this approximation is valid when the mean free path is significantly smaller than the characteristic length scale of the problem. This is typically the case at atmospheric pressures and length scales of the order of millimeters or greater [41]. In other words, as long as the thermal velocity is comparable to the drift velocity and the continuum regime is applicable (Knudsen number $K n$ is low, $\lambda / L=K n \ll 1$, as the mean free path $\lambda$ at atmospheric conditions is $O\left(10^{-7} \mathrm{~m}\right)$ and the actuator characteristic length $L$ is $\left.O\left(10^{-3} \mathrm{~m}\right)\right)$, the inertial components in the momentum equation can be neglected. Such approximation yields a balance between the collision/ionization effects and the drift-diffusion components, instead of the full momentum equation. Thus the fluxes of charged particles are given by:

$\vec{\Gamma}_{k}=n_{k} \vec{v}_{k}= \pm \mu_{k} n_{k} \vec{E}-D_{k} \nabla n_{k}+n_{k} \vec{v}_{b}$

In Eq. (1), the drift-diffusion flux consists of a mobility flux term (for charged species), a diffusive flux term, and the species flux due to the flow velocity field.

The simplest set of equations within the fluid model framework, containing the basic physics necessary for gaseous plasma discharges, is the continuity equations for electrons, positive ions and negative ions (to account for the development of the spacecharge), coupled with Poisson's equation (to account for the modification of the electric field due to space-charge), which is summarized in Table 1.

\subsection{Transport properties}

The production and loss terms in the continuity equations of the charged particles include different reactions. If we assume that the working fluid is air, composed of $\mathrm{N}_{2}$ and $\mathrm{O}_{2}$ only, then the following reactions are considered. 
Table 1

Summary of the governing equations.

\begin{tabular}{ll}
\hline & Equations \\
\hline Continuity equations: & \\
Electron; & $\frac{\partial n_{e}}{\partial t}+\nabla \cdot \vec{\Gamma}_{e}=S_{e}-L_{e}$ \\
& $S_{e}=\alpha\left\|\vec{\Gamma}_{e}\right\|$ \\
& $L_{e}=r_{e p} n_{e} n_{p}+\eta\left\|\vec{\Gamma}_{e}\right\|$ \\
& \\
Positive ions; & $\frac{\partial n_{p}}{\partial t}+\nabla \cdot \vec{\Gamma}_{p}=S_{p}-L_{p}$ \\
& $S_{p}=\alpha\left\|\vec{\Gamma}_{e}\right\|$ \\
& $L_{p}=r_{e p} n_{e} n_{p}+r_{n p} n_{n} n_{p}$ \\
Negative ions & $\frac{\partial n_{n}}{\partial t}+\nabla \cdot \vec{\Gamma}_{n}=S_{n}-L_{n}$ \\
& $S_{n}=\eta\left\|\vec{\Gamma}_{e}\right\|$ \\
& $L_{n}=r_{n p} n_{n} n_{p}$
\end{tabular}

Momentum equations

$$
\begin{aligned}
& \vec{\Gamma}_{e}=n_{e} \vec{v}_{e}=-\mu_{e} n_{e} \vec{E}-D_{e} \nabla n_{e}+n_{e} \vec{v}_{b} \\
& \vec{\Gamma}_{p}=n_{p} \vec{v}_{p}=\mu_{p} n_{p} \vec{E}-D_{p} \nabla n_{p}+n_{p} \vec{v}_{b} \\
& \vec{\Gamma}_{p}=n_{p} \vec{v}_{p}=\mu_{p} n_{p} \vec{E}-D_{p} \nabla n_{p}+n_{p} \vec{v}_{b}
\end{aligned}
$$

Poisson equation:

$$
\nabla .(\varepsilon \nabla \varphi)=-e\left(n_{p}-n_{e}-n_{n}\right)-\delta_{s} \sigma
$$

\section{- Ionization}

$e+\mathrm{N}_{2}\left(\mathrm{O}_{2}\right) \rightarrow 2 e+\mathrm{N}^{+}{ }_{2}\left(\mathrm{O}^{+}{ }_{2}\right)$

- dissociative electron-ion recombination,

$$
\begin{aligned}
& e+\mathrm{O}^{+}{ }_{2} \rightarrow \mathrm{O}+\mathrm{O} \\
& e+\mathrm{O}^{+}{ }_{4} \rightarrow \mathrm{O}_{2}+\mathrm{O}_{2}
\end{aligned}
$$

- Ion-ion recombination

$$
A^{+}+B^{-}+M \rightarrow A+B+M
$$

- dissociative electron attachment and detachment

$$
\begin{aligned}
& e+\mathrm{O}_{2} \rightarrow \mathrm{O}^{-}+\mathrm{O} \\
& \mathrm{O}^{-}+\mathrm{N}_{2} \rightarrow e+\mathrm{N}_{2} \mathrm{O}
\end{aligned}
$$

In the above equations the ionization coefficient $\alpha$ needs be defined; it measures the number of ionization by electron per unit distance. In other words, it is a measure of energy gain by the charged particles between collisions. The dissociative electron-ion recombination is the fastest mechanism of the bulk recombination of the weakly ionized gas. The rate is given as $-r_{e p} n_{e} n_{p}$. At low pressure environment the ion-ion recombination process takes place through binary collisions and the reaction is similar to charge transfer, and at moderate pressures the reaction proceeds through triple collisions. The rate of ion-ion recombination is also given as $r_{n p} n_{n} n_{p}$. The electron attachment is the formation and depletion of negative charged ions in the partially ionized air. The electron detachment is the main mechanism of removing electron from negatively charged ions. This loss of electron number density is given by $-v_{a} n_{e}$, where $v_{a}$ is the attachment frequency. However, for the sake of stability of the numerical simulation, the rate of attachment is defined in a similar way to the ionization rate, as $\eta\left\|\vec{\Gamma}_{e}\right\|$.

An accurate gas discharge plasma modeling requires an extensive knowledge of the transport parameters of the gas in question. The fluid model uses transport parameters found from the kinetic model or from experimental results. In the former approach, the parameters are obtained by solution of the stationary Boltzmann equation. However, we should mention that there are deviations between different correlations reported in the literature, which may affect the results significantly. The gas properties such as transport coefficients (momentum transfer rate such as $\mu$, and $D$ ), collisional ionization coefficient $\alpha$ and energy loss rates may be obtained simply by considering the local-field approximation(LFA) [42]. This implies that the dependence of these coefficients on the mean energy is supposed to be the same as at equilibrium. The local equilibrium assumption implies that the transport coefficients depend on space and time only through the local value of the electric field $\vec{E}(x, t)$ (functions of the reduced electric field $E / N$ where $E$ is the field amplitude and $N$ the gas number density [41]). In this way, the transport coefficients are assumed to be the same as those which could be measured or calculated under a uniform and constant electric field $\vec{E}$ (hydrodynamic regime). Essentially this means that the electron distribution function is in local equilibrium with the neutral plasma. This assumption is valid as long as the relaxation time for achieving a steady state electron energy distribution function is short compared with the characteristic time of discharge development. To obtain the properties of electron transport such as mobility, diffusivity, mean energy, etc., as a function of $E / N$, the solution of a steady state Boltzmann equation is considered, under constant field condition, to obtain the electron energy distribution function in the gas under investigation. For most of the electron-induced processes the reaction rates are calculated on the basis of energy-dependent cross-sections with a separate program, called BOLSIG [43]. This program is applied for a wide range of different, fixed reduced electric fields and gives the dependence of the rates of ionization, dissociation, and excitation of the particles in plasma discharges as a function of the reduced electric field $E / N$ by solving the Boltzmann kinetic equation in a homogeneous field with uniform and steady conditions. In this study, rate constants of the different electron-induced processes in the above reactions and the transport coefficients, including mobility of the electrons (the corresponding diffusion coefficients are then calculated using Einstein's relation), were obtained with the BOLSIG Solver or were extracted from [37] and are presented in Table 2.

\subsection{Boundary and initial conditions}

\subsubsection{Initial condition}

The initial condition to start the simulation requires specifying the starting number densities for the various species. The value of $n_{0}$ is used as a reference value (and also as a measure of pre-ionization of the neutral gas) to define the initial values. Thus, the initial positive ion and electron number densities are assumed to be uniform and equal to $n_{0}$ in the plasma domain, while the negative ion charge density is assumed to be zero.

\subsubsection{Electric potential boundary conditions}

The boundary value of the electric potential is defined at the two electrodes (anode and cathode) using a Dirichlet boundary 
Table 2

Summary of property models employed for discharge simulation.

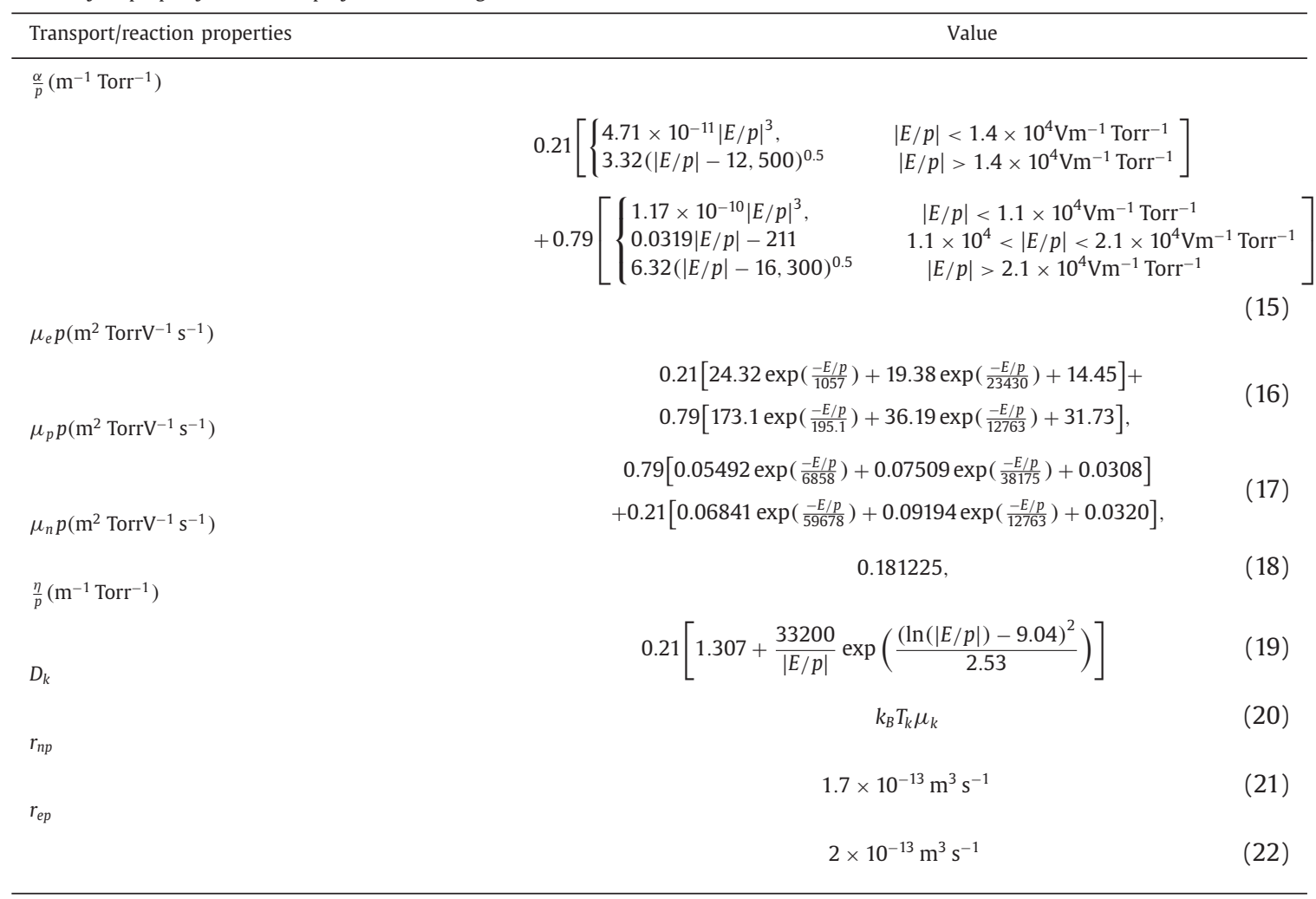

condition as follows;

$\varphi=V_{0} f(t) \quad$ at anode,

$\varphi=0 \quad$ at cathode,

$f(t)$ is a function representing the time-dependent voltage waveform. A homogeneous Neumann boundary condition is applied for the electrostatic potential at the open boundaries (far field):

$\frac{\partial \varphi}{\partial n}=0 \quad$ at far field,

In addition, the boundary conditions for the electric field and electric potential at the dielectric barrier and plasma interface prescribe a continuous tangential component of $\vec{E}$ and a jump of the normal component due to the presence of localized electric charge. Considering the accumulated surface charges, and based on Gauss's divergence law for the electric displacement and Faraday's induction law [23], these boundary conditions are written as:

$\vec{n} \times\left(\vec{E}-\vec{E}_{d}\right)=0$

$\varepsilon_{0} \vec{E} \cdot \vec{n}-\varepsilon_{d} \varepsilon_{0} \vec{E}_{d} \cdot \vec{n}=\sigma$

In the above equations, $\varepsilon_{0}$ is the permittivity of the space, $\varepsilon_{d}$ is the relative permittivity of the dielectric layer, $\vec{E}$ and $\vec{E}_{d}$ are respectively the electric field in the gas and the dielectric, and $\sigma$ is calculated by integrating the net charge density over the surface and has the dimension of $\mathrm{C} / \mathrm{m}^{2}$. This charge accumulation on the surface is considered as the result from instantaneous recombination of the charged particles after satisfying the imposed boundary conditions for the charged species, includes accumulation of both positive and negative charges $\left(\sigma=\sigma_{+}+\sigma_{-}\right)$and is computed using the flux of charged species to the surface [22] as:

$\sigma=\int_{0}^{t}-e\left(\vec{\Gamma}_{p}-\vec{\Gamma}_{e}-\vec{\Gamma}_{n}\right) \cdot \vec{n} d t=\int_{0}^{t}-\vec{j} \cdot \vec{n} d t$ where $\vec{j}$ is the current due to fluxes of charged particles. We note that, in view of the relatively short duration of the micro-discharge compared to the microsecond time scale of the AC cycle, the instantaneous surface recombination is an acceptable approximation. Eq. (26) states that that the tangential electrical field strength is continuous across the media interface. For a Cartesian grid, this means that the rate of change for electric potential in $z$ must be identical across the interface along the $x$ coordinate and the rate of change of electrical potential in $x$ must be equal along the $z$ coordinate across the media interface. This requirement is automatically satisfied by the two-dimensional formulation.

The second condition expressed by Eq. (27), is the balance of the discontinuity of the normal component of the electric displacement across the plasma/dielectric interface by the net surface charge density on the interface by emission, desorption, and accumulation. This condition is independent of all the chemicalphysical processes at the interface. This equation could be rewritten by considering the electric potential as:

$\left.\varepsilon_{0} \frac{\partial \phi}{\partial n}\right|_{\text {air }}-\left.\varepsilon_{0} \varepsilon_{d} \frac{\partial \phi}{\partial n}\right|_{d}=\sigma$

In other words, the current continuity is enforced by equating the drift current and the displacement current in the fluid domain to the displacement current inside the dielectric material [44].

\subsubsection{Charge number density boundary conditions}

At the domain boundary away from the dielectric/electrode surface, incoming/outgoing flux of particles is negligible. Thus a zero normal gradient boundary condition is assigned (i.e. the slopes of the solution variables are equal to zero) which assumes that impact far away from the fluid-actuator interface is insignificant. This boundary condition is expressed as:

$$
\begin{aligned}
& \vec{\Gamma}_{k} \cdot \vec{n}=0, \\
& \frac{\partial n_{k}}{\partial n}=0,
\end{aligned}
$$


Boundary conditions for the charged particle number density are given on the electrode and dielectric layer surface through a balance between the fluxes of the charge particles absorbed by the surface and the fluxes reflected from the surface. When the surfaces are in anodic behavior (e.g. exposed electrode as anode and $\sigma>0$ ), the incident positive ion fluxes at the surface is collected considering thermal equilibrium, while the electron fluxes are reflected from the surface. In this case, the electron and negative ion fluxes are also based on the electron thermal velocity $\left(v_{t h}=\sqrt{8 k_{B} T /(\pi m)}\right)$, and have magnitudes given by $\Gamma_{t h}=\frac{n v_{t h}}{4}$. Those fluxes are directed towards the wall and it is implied that the hydrodynamic flux equals the flux from the dielectric surface:

$\vec{\Gamma}_{p}=-\frac{1}{4} n_{p} v_{t h, p}$

$\vec{\Gamma}_{n}=-\mu_{n} n_{n} \vec{E}-\frac{1}{4} n_{n} v_{t h, n}$

$\vec{\Gamma}_{e}=-\mu_{e} n_{e} \vec{E}-\frac{1}{4} n_{e} v_{t h, e}$

In a similar way, when the exposed electrode is a cathode and the dielectric barrier is in cathodic behavior $(\sigma<0)$, positive ion fluxes are reflected from the surface and thus the positive ion flux will be equal to its drift part. For the electrons and negative ions, we assume that their fluxes are collected at the surface and thus:

$\vec{\Gamma}_{p}=\mu_{p} n_{p} \vec{E}-\frac{1}{4} n_{p} v_{t h, p}$

$\vec{\Gamma}_{n}=-\frac{1}{4} n_{n} v_{t h, n}$

$\vec{\Gamma}_{e}=-\frac{1}{4} n_{e} v_{t h, e}$

These boundary conditions (Eqs. (31-36)) can be written in a compact form as follows:

$\vec{\Gamma}_{p} \cdot \vec{n}=\min \left(\mu_{p} n_{p} \vec{E} \cdot \vec{n}, 0.0\right)-\frac{1}{4} n_{p} v_{t h, p}$

$\vec{\Gamma}_{n} \cdot \vec{n}=\min \left(-\mu_{n} n_{n} \vec{E} \cdot \vec{n}, 0.0\right)-\frac{1}{4} n_{n} v_{t h, n}$

$\vec{\Gamma}_{e} \cdot \vec{n}=\min \left(-\mu_{e} n_{e} \vec{E} \cdot \vec{n}, 0.0\right)-\frac{1}{4} n_{e} v_{t h, e}$

The above equations are in fact preventing charged particle fluxes at the wall when the driving force is directed towards the wall. In Eqs. (37) and (38), the second term is the thermal flux of ions to the wall and the first term is a drift flux contribution due to the sheath potential. This term gives a nonzero drift flux for positively charged ions in a positive sheath (i.e., decreasing potential toward the wall) and zero flux in the same sheath for negatively charged ions. The opposite is true for negative sheaths.

When positive ions hit the cathode (either the electrode surface or the dielectric surface), it releases $\gamma_{\text {sec }}$ secondary electron(s). The secondary emission coefficient $\gamma_{\text {sec }}$ is generally between 0 and 1 . Its value depends on the particle, its energy, the surface material and temperature. In this case, the expression of the electron flux due to secondary emission is:

$\vec{\Gamma}_{\text {sec }}=\gamma_{\text {sec }} \vec{\Gamma}_{p}$

Thus the total electron flux at the wall (Eq. (39)) becomes,

$\vec{\Gamma}_{e} \cdot \vec{n}=\min \left(-\mu_{e} n_{e} \vec{E} \cdot \vec{n}, 0.0\right)-\frac{1}{4} n_{e} v_{t h, e}-\gamma_{\sec } \vec{\Gamma}_{p} \cdot \vec{n}$

This boundary condition is essential because the mechanism and speed of the streamer breakdown process depend on the avail- ability of these secondary electrons. As mentioned in [45], this implementation of the secondary emission is not consistent with the experimental value of $\gamma_{s e c}$ at the cathode. In experiments, $\gamma_{\sec }$ is calculated as the ratio between the flux of secondary electrons and the particle flux to the electrodes. As a solution, thermal velocity of zero at the cathode is imposed. However, due to the variation of the plasma potential responding to the applied voltage, it is fairly delicate to detect which wall represents the instantaneous cathode. An alternative solution is suggested in $[46,47]$.

Here for simplicity, we assume that the thermal velocity is zero on all surfaces. As mentioned before, variation of the potential causes the drift flux of electrons to change along the surface leading to a jump of the electron concentration at a point where $E=0$. In some cases, this creates numerical problems because the steep gradients of the solution cannot be resolved in the vicinity of the jump. Hence to determine the instantaneous cathode and ensure a smooth and stable variation of the charge boundary condition on the surface, the exact solution of the boundary conditions mentioned above is obtained and the boundary condition is implemented as follows:

$$
\begin{aligned}
& n_{p}=e^{\frac{\max \left(\vec{u}_{p} \cdot \vec{n}_{0.00) \Delta}\right.}{-D_{p}}}\left(n_{p}^{I n t}\right) \\
& n_{n}=e^{\frac{\max \left(\vec{u}_{n} \cdot \vec{n}_{0} .0 .0\right) \Delta}{-D_{n}}}\left(n_{n}^{\operatorname{Int}}\right) \\
& n_{e}=\frac{\gamma n_{p} \mu_{p}}{\mu_{e}} \times\left(1-e^{\frac{\max \left(\vec{u}_{e} \cdot \vec{n}_{0}, 0.0\right) \Delta}{-D_{e}}}\right)+e^{\frac{\max \left(\vec{u}_{e} \cdot \vec{u}_{e}, 0.0\right) \Delta}{-D_{e}}}\left(n_{e}^{\text {Int }}\right)
\end{aligned}
$$

The above equations are general boundary conditions and are valid at any surface.

\subsection{Interaction of the discharge and the flow}

The problem can be separated into two parts. One of them is modeling of plasma phenomena (ionization, recombination, evolution of electric field, etc.) that occur on a sub nanosecond-tomicrosecond time scale on the background of "frozen" flow field. The second problem consists in coupling the plasma with the flow by means of plasma-induced force and heating rate. In the first approach, the coupling between the plasma solver and the flow solver is one way, that is, a gas heating and source term is imposed to the plasma solver and the plasma is only influencing the flow field through the electro-hydrodynamic force or gas heating. The electro-hydrodynamic (EHD) force associated with the momentum transfer from charged particles to neutral molecules in the volume above the dielectric layer is given by [19]:

$\tilde{\mathrm{f}}=\sum_{k=p, e, n} \frac{\overrightarrow{j_{\mathrm{k}}}}{\overrightarrow{\mu_{\mathrm{k}}}} \approx e\left(n_{p}-n_{e}-n_{n}\right) \vec{E}$

This equation thus provides the total force per unit volume acting on the neutral molecules and is equal to the Coulomb force acting on the charged particles. In other words, the momentum gained by the charged particles in the electric field is exactly and locally balanced by collisions, and entirely transmitted to neutral molecules.

When the plasma equations are solved sequentially with an external flow model, the gas heating source terms are collected and communicated to the flow model which is then responsible for determining the bulk temperature [3]. The gas heating source terms are calculated by the plasma solver and include the ion Joule heating term due to the work done on the ions by the electric field, inelastic collisional heating term due to the quenching of electronically excited species such as nitrogen, oxygen metastables, and the elastic collisional heating term due to elastic electron impacts with the background gas. These gas heating source terms are important for atmospheric DBD plasma actuator driven by a nanosecond voltage. 
In this paper, for the sake of simplicity, the one-way coupling of the plasma solver and the flow solver is adopted. We should mention that this is a valid assumption in a large range of applications, due to the large difference of the fluid characteristic time scales and the time scales of operating plasma dynamics.

However, when the plasma solver and the flow solver are communicating in two-way coupling, the flow influences the plasma as a momentum source for the species. This momentum source enters in the drift-diffusion equations. This effect is particularly important for the neutral species, as there are no electric forces to modify their speed. Moreover, if the fluid dynamic time scales become comparable to the plasma time scales, then the two-way coupling will need to be handled and the fluid solution advancement should be performed using the DBD time scale.

\section{Numerical procedures}

The coupled plasma-fluid problem is inherently nonlinear and exhibits wide ranges of time and length scales. Numerical solution of the charged particles governing equation coupled with Poisson equation is subjected to serious limitations in the selection of numerical time step. Sufficiently small time steps provide stable and accurate solution, however the computational run time will be massively high for the purpose of simulation. Thus, the approach selected for treating the time discritization and advancing the time step should be capable of both providing the required accuracy and stability while providing a reasonable computational cost. In this view, different approaches could be found in the literatures which have been tested to reach this purpose. Moreover, there are different time scales in the plasma formation, originating from fast ionization and slow recombination processes along with drift and diffusion of the species, which typically lead to a stiff problem due to the vastly differing time scales. Moreover, high accuracy may be required for all time scales. To resolve this issue, special treatments need to be considered for solving the governing equation of the plasma dynamics some possible remedies could be:

- Implicit methods are favored for stiff problems. They are often used but may be expensive and difficult to derive. Marching the solution in time will typically need to be implicit because of the diffusion terms, which otherwise would cause a severe time step restriction. The reaction terms may also contribute to the need of using an implicit method if some fast reactions reach equilibrium on a much faster time scale than the processes being modeled (i.e., if the reaction terms are "stiff").

- Operator splitting $[6,39,48]$ (or fractional step method) treats the separate processes independently, i.e. solves the homogenous (convective part) and the inhomogeneous (source terms) part of the equations separately. Although splitting allows each component to be modeled efficiently, none should be allowed to vary significantly before interacting with others. In this case, the simulation could take advantage of the best scheme that can be chosen in each case (which is useful when some terms are insignificant). Moreover, accuracy and stability issues can be dealt with at each stage (aiding robustness) and also it is relatively simple to deal with processes acting on widely differing time scales, due to flexibility in the choice of time-stepping. This is relatively simple, cheap to run and fairly stable. But it also ignores the fact that the processes are coupled and do interact, so it may misinterpret the results. Formally, the scheme is only first order accurate.

- Sub-cycling or dual time-stepping is used as a simpler alternative, particularly when the source terms are complicated and highly nonlinear. This uses many steps of an explicit method to reach the same time level as the global time step.
- Adaptive time-stepping might be desirable allowing larger time steps when it is possible.

Although these remedies could benefit the solution procedure, they all require that the dependent variables do not change dramatically within a global time step. Moreover, small time steps are required to resolve the effects of rapid transient phenomena. But, larger time steps may be necessary to allow the simulation to be run within a reasonable time. In other words, numerical stability governs the size of the time step which can be used to get any results at all. In general, the time step is constrained by accuracy which must also be balanced against efficiency and stability. In the following sections, some of the numerical treatments used in this paper for the solution of the governing equations of plasma-fluid model are presented.

\subsection{Temporal discretization}

The plasma/flow interaction is fundamentally a multi-scale problem [6] with a large range of time scales, from shorter than the pico-second (e.g. dielectric relaxation time) to time scale of the airflow [10]. In the present study, we have employed an operatorsplitting sequential solution algorithm. In using time-split algorithms for processes operating in a wide range of time scales, the choice of time step size is typically determined by the smallest time scale, but need not necessarily be chosen as such. To speed up the solution procedure, an adaptive intermediate time scale is chosen to advance the overall system in time.

\subsubsection{The fractional step method and sub-cycling}

The continuity equations of the electron, positive and negtive ions (Eqs. (2)-(4)) can be handled by the fractional step method. A similar procedure was adopted in [44] for modeling DBD plasma actuators. To this purpose, the main problem needs to be split in two sub-problems. One issue regarding the solution of the continuity of the charged particles arises due to the presence of very fast chemical rates, and in particular by large decay rates, which makes the coupling of source terms with the drift diffusion terms quite unstable. Therefore, we will treat the terms separately by rewriting the governing equation as follows;

$\frac{\partial n_{k}}{\partial t}=\mathrm{H}\left(n_{k}\right)+\mathrm{R}\left(n_{k}\right)$,

and splitting them into;

$\frac{\partial n_{k}}{\partial t}=\mathrm{H}\left(n_{k}\right)$,

$\frac{\partial n_{k}}{\partial t}=\mathrm{R}\left(n_{k}\right)$

Two approaches may be considered to deal with the above equations. In the first, Eq. (47A) is solved with a time step $\Delta t$, and then the result will be used to solve Eq. (47B) with the same time step $\Delta t$.

This approach is often known as Godunov splitting and essentially needs two sub-cycles. However, it is possible to use more cycles using an even number of overall steps while keeping the order in which the solution of Eq. (47A) and Eq. (47B) are carried out. An alternative approach which requires three sub-cycles is the Strang splitting [49]. In this case,

- Eq. (47A) is solved with time step $\Delta t / 2$,

- Eq. (47B) is solved with time step $\Delta t$,

- Eq. (47A) is solved again with time step $\Delta t / 2$.

The small modification in Strang splitting allows the overall scheme to be second order accurate. It is also possible to use more sub-cycles. In such case, the first $\Delta t / 2$ step (Eq. (47A)) is followed by a series of $\Delta t$ steps (Eq. (47B)) and finishing with one $\Delta t / 2$ step. 
The Strang splitting is a symmetric version of this more general procedure. Another variant of Strang splitting method is obtained by splitting up the solution procedure into independent steps corresponding to the advection, diffusion and reaction processes, and handling them independently at each step [50]. A similar methodology that uses both explicit and implicit formulation of the advection and diffusion part is described in [51].

\subsubsection{Adaptive time-stepping}

As mentioned before, for accuracy and stability of the numerical scheme, the time step is defined by the CFL condition, i.e. particles cannot move more than a grid size during the time step. The modified or adaptive time step concept is based on an estimation of the onset of the breakdown. In this way, the time step is defined by electron velocities, considering a threshold, based on maximum electron number density for onset of breakdown [52].

\subsubsection{Super-time-stepping}

As noted above, explicit methods are easy to implement but have very restrictive time step constraints. Implicit solution methods can be unconditionally stable but have the disadvantage of being computationally costly or difficult to implement. Recently, super-time-stepping methods [53] for treating the unsteady terms were developed and occupy an intermediate position. In such methods each super step takes $s$ explicit Runge-Kutta like time steps to advance the parabolic terms by a time step that is larger than a single explicit time step. Meyer et al. [54] derived first and second order super-time-stepping schemes based on the RungeKutta-Legendre (RKL) methods. The general recursion formula of first order (RKL2) and second order RKL (RKL4) are respectively as follows:

$$
\begin{aligned}
& n^{0}=n^{t} \\
& n^{1}=n^{0}+\tilde{\mu}_{1} \Delta t \mathrm{P}\left(n^{0}\right), \\
& n^{j}=\bar{\zeta}_{j} n^{j-1}+\bar{\zeta}_{j} n^{j-2}+\tilde{\mu}_{j} \Delta t \mathrm{P}\left(n^{j-1}\right), \quad 2 \leq j \leq s \\
& n^{t+\Delta t}=n^{j}
\end{aligned}
$$

where

$$
\tilde{\mu}_{j}=\frac{2 j-1}{j} \frac{2}{s^{2}+s}, \quad \bar{\zeta}_{j}=\frac{2 j-1}{j}, \quad \bar{\zeta}_{j}=\frac{1-j}{j}
$$

and

$$
\begin{aligned}
& \begin{array}{l}
n^{0}=n^{t}, \\
n^{1}=n^{0}+\tilde{\mu}_{1} \Delta t \mathrm{P}\left(n^{0}\right), \\
n^{j}=\bar{\zeta}_{j} n^{j-1}+\bar{\zeta}_{j} n^{j-2}+\left(1-\bar{\zeta}_{j}-\bar{\zeta}_{j}\right) n^{0}+\tilde{\mu}_{j} \Delta t \mathrm{P}\left(n^{j-1}\right) \\
\quad+\tilde{\gamma}_{j} \Delta t \mathrm{P}\left(n^{j-1}\right), \quad 2 \leq j \leq s \\
n^{t+\Delta t}=n^{j}
\end{array} \\
& \text { where } \\
& \tilde{\mu}_{j}=\frac{(2 j-1)(j+2)(j-1)^{2}}{j(j-2)(j+1)^{2}} \frac{4}{s^{2}+s-2}, \\
& \bar{\zeta}_{j}=\frac{(2 j-1)(j+2)(j-1)^{2}}{j(j-2)(j+1)^{2}}, \quad \bar{\zeta}_{j}=\frac{(j-1)^{3}\left(j^{3}-4\right)}{j^{3}(j+1)(j-3)} \\
& \tilde{\mu}_{1}=\frac{4}{3\left(s^{2}+s-2\right)}, \quad \tilde{\gamma}_{j}=\frac{(2 j-1)(j+2)(j-1)\left(j^{2}+j-2\right)}{2 j^{2}(j-2)(j+1)^{2}}
\end{aligned}
$$

The above described super-time-stepping method can be used to solve the charged particles conservation equation. However, to be used for advection-diffusion problems, the equations need to be solved in operator splitting manner. The number of stages of RLK2 may be chosen as the lowest odd value that satisfy the stability criteria mentioned in [54]. Hence, the continuity equations of the number density of the charged particles will be divided in two sub-problems, considering the homogenous (transport terms) and non-homogenous (source terms) operators as in Eq. (46).

In this way, an entire time step is completed by taking a halflength time step using s-stage super-time-stepping strategy for the operator $\mathrm{H}$, and then a full length time step explicitly for the operator $\mathrm{R}$, and finally a second half-length step using s-stage supertime-stepping. An entire, time step, representing the action of all operators, contains the following stages:

- Solve $\left(n_{k}^{t+\Delta t}\right)^{*}=\mathrm{H}(\Delta t / 2)+n_{k}^{t}$ over time $\Delta t / 2$ with data $n_{k}^{t}$ to obtain $\left(n_{k}^{t+\Delta t}\right)^{*}$

- Solve $\left(n_{k}^{t+\Delta t}\right)^{* *}=\mathrm{R}(\Delta t)+\left(n_{k}^{t}\right)^{*}$ over time $\Delta t$ with data $\left(n_{k}^{t+\Delta t}\right)^{*}$ to obtain $\left(n_{k}^{t+\Delta t}\right)^{* *}$

- Solve $n_{k}^{t+\Delta t}=\mathrm{H}(\Delta t / 2)+\left(n_{k}^{t}\right)^{* *}$ over time $\Delta t / 2$ with data $\left(n_{k}^{t+\Delta t}\right)^{* *}$ to obtain $n_{k}^{t+\Delta t}$

\subsubsection{Semi-implicit solution of Poisson and transport equations}

When time integration of the Poisson-transport system is treated explicitly, the Poisson equation and the charged particles transport equations are solved successively, while the electric field calculated at time $t^{k}$ is supposed to be constant during the integration of the charged particles transport equations between times $t^{k}$ and $t^{k+1}$. However, the strong coupling between the charge density equations and the electric potential results in a severe time step restriction for explicit methods (the time step must be smaller than the dielectric relaxation time, the so-called "dielectric relaxation time step constraint"). In order to overcome this constraint, the Poisson equation is solved in a semi-implicit manner for the electric potential [55]. Semi-implicit treatment of the Poisson equation has been proven to provide stable results and allow larger time steps (similar approach were used by $[39,48]$ ). To derive the semi-implicit version of the Poisson equation, the species number densities in the source term of the Poisson equation need to be linearized implicitly. This is accomplished by substitution of the charges number density in the source term using the species transport equations, and expanding the right hand side with a first order Taylor series expansion:

$$
\begin{aligned}
& \nabla .\left(\varepsilon \nabla \varphi^{t+1}\right)=-e\left(n_{p}{ }^{t+1}-n_{e}{ }^{t+1}-n_{n}{ }^{t+1}\right)=\rho_{c}^{t+1}=\rho_{c}^{t}+\rho_{c}^{\Delta t} \\
& =-e\left(n_{p}{ }^{t}+\Delta t \frac{\partial n_{p}}{\partial t}-n_{e}{ }^{t}-\Delta t \frac{\partial n_{e}}{\partial t}-n_{n}{ }^{t}-\Delta t \frac{\partial n_{n}}{\partial t}\right)
\end{aligned}
$$

Using the species conservation equations and treating the terms containing drift velocities (thus electric field), the Poisson equation can be rewritten as,

$$
\begin{aligned}
\nabla . & \left(\left[\varepsilon+\Delta t\left(\mu_{p} n_{p}+\mu_{e} n_{e}+\mu_{n} n_{n}\right)\right] \nabla \varphi\right) \\
= & -e\left(n_{p}{ }^{t}+\Delta t \nabla .\left(\nabla n_{p}{ }^{t}\right)-\nabla .\left(n_{p} \vec{u}\right)-n_{e}{ }^{t}-\Delta t \nabla .\left(\nabla n_{e}{ }^{t}\right)\right. \\
& \left.+\nabla .\left(n_{e} \vec{u}\right)-n_{n}{ }^{t}-\Delta t \nabla .\left(\nabla n_{n}{ }^{t}\right)+\nabla .\left(n_{n} \vec{u}\right)\right)
\end{aligned}
$$

The charge continuity equations are also solved with a semiimplicit method. In this way, the flux terms are evaluated with the value of charge densities at new time step, while the source terms (ionization, recombination, and attachment) are estimated with charge densities from the previous time step. Semi-implicit treatment of the Poisson and transport equations could be attractive in terms of computation time when using larger time steps compared to the stability constraint (CFL or Maxwell relaxation time). However, such scheme is only first order in space and said to be quite diffusive. On the other hand, fully explicit second order Poisson/transport coupling is feasible but at prohibitive computational cost.

\subsection{Implementation of the model}

The coupled systems of species equations (Eqs. (2)-(7)) as well as Poisson's equation (Eq. (8)) are solved using OpenFOAM. As 
Coupled Boundary

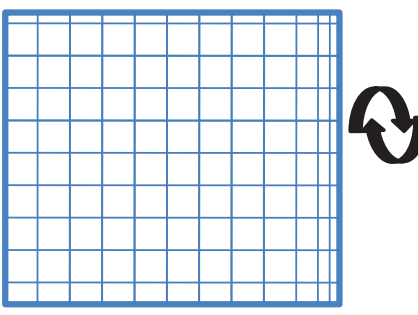

Region 1

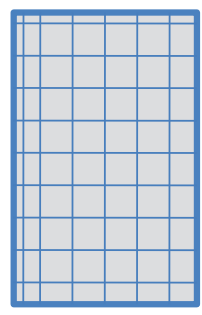

Region 2

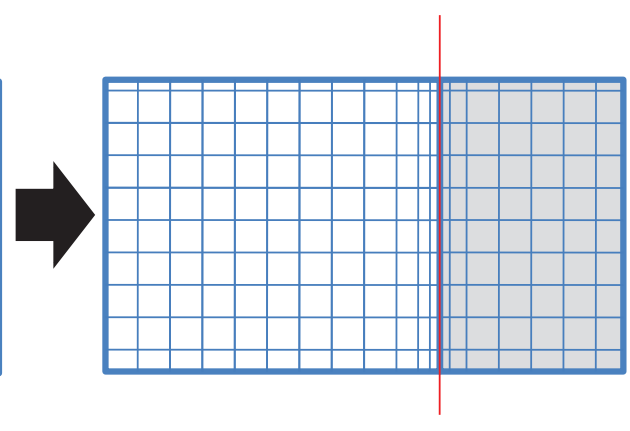

Region 1+2

Fig. 1. A multi-region coupled domain for electric potential.

mentioned in [21], The 2D Cartesian geometry can provide realistic results if the plasma is not filamentary in the direction perpendicular to the simulation plane or if the width of the filaments in this direction is large with respect to the thickness of the filament above the dielectric surface.

The implementation of the plasma-fluid model needs some extra treatment regarding the multi-region coupled solution of the Poisson equation and inter-coupling between the transport equations and the electric field, which are described in the next sections.

\subsubsection{Multi-region coupling of the electric potential}

The presence of the dielectric layer brings the need of the solution of the electric potential equation in the gas (e.g. air) and the solid domains (e.g. dielectric layer). In Fig. 1, a schematic of the multi-region problem is shown. To be able to obtain the solution of the Poisson equation in multiple regions, a multi-region solver is developed based on the partitioning approach.

In the partitioned approach, the governing equations (Poisson equation) are solved in each zone separately with the appropriate boundary conditions using a segregated solver. In other words, the iterative solution is obtained region by region, and the interface boundary conditions are treated also in a segregated manner, where one of the interface equations is applied as a boundary condition to one sub domain and the other boundary condition to the second sub domain. These boundary conditions are implemented using a mixed type BCs for PDE on different parts of the boundary of the domain of the equations. In this way, the resulting conditions are responsible for coupling between different regions and have to be used for each region according to the updated values in the neighboring region. The schematic of the partitioned approach for the plasma-fluid problem is illustrated in Fig. 2.

In this approach the convergence is assured by using a PIMPLE like plasma loop for solution of the governing equations. In other words, for each time step, given a maximum number of iterations, the governing equations will be solved alternatively for every cell of the coupled regions and when the convergence is reached, the solution will be proceeded to the next time step.

\subsubsection{Coupling of Poisson and charged particles transport}

In the plasma model, there are two issues regarding the coupling of the variables. First, there is the coupling of the number density of the particles and electric potential, and the second is related to the multi-region solution of the Poisson equation. We used the partitioned approach for solving the Poisson equation. In this way, separate governing equations will be solved by solving separate matrix equation systems, coupled at the boundary interface, and then sub-iterate until coupled convergence is reached. In this context, a PIMPLE like algorithm is used to guarantee the coupling between the electrical potential and charged particles density equation and the coupling at the dielectric interface of solid zone (dielectric material) and fluid zone (air). This iterative solution of the discretized equations in each time step with sufficient internal iterations will guarantee enhanced coupling between the dependent variables. The schematic of the algorithm is shown in Fig. 3. The working steps (The iterative procedure of the PIMPLE like plasma loop) for a generic i-th iteration are as follows:

(i) At the beginning of each time step, the values of charge number densities and transport coefficients are known, and they will be used to solve the Poisson equation. The governing equations for the electric field in the fluid region are solved with the interface boundaries conditions on the basis of the values estimated at the previous iteration $(i-1)$-th.

(ii) When the electric field is updated, all the transport properties and source terms of the charge continuity equations will be updated according to the new value of the electric field.

(iii) Continuity equations for the charge densities will be solved with updated values of the electric field, source terms and the interface boundary conditions on the basis of the values estimated at the previous ( $i-1)$-th. Then, the source term and artificial permittivity arising due to the semi-implicit treatment of the Poisson equation will be recalculated.

(iv) The governing equations of electric field in the solid phase is solved with the interface boundary conditions on the basis of the new values of new estimated electric field in the fluid region and on the basis of the electric field in the solid region estimated at $(i-1)$-th iteration. Moreover, the surface charge density will be corrected also at this step.

(v) Convergence check the new fluid and solid fields at gas/solid interface boundaries by means of the absolute and relative residuals; if convergence is not reached the procedure restarts.

(vi) If the convergence criteria are satisfied, the solver computes the gas dynamics. Then this solution is used as starting point for the next iteration.

\subsection{Grid requirements}

The plasma processes are very sensitive to the local electric field, which in the DBD case varies on a characteristic scale of the order of 10 microns for high applied voltages. The necessity of the correct resolution of the plasma dynamics leads to a grid size of the order of several microns. However, the increase of the operating voltage leads to the further decrease of the grid size. Thus, the computational grid needs to be selected based on the outcome of testing several grid sizes to ensure enough resolution of the steep 


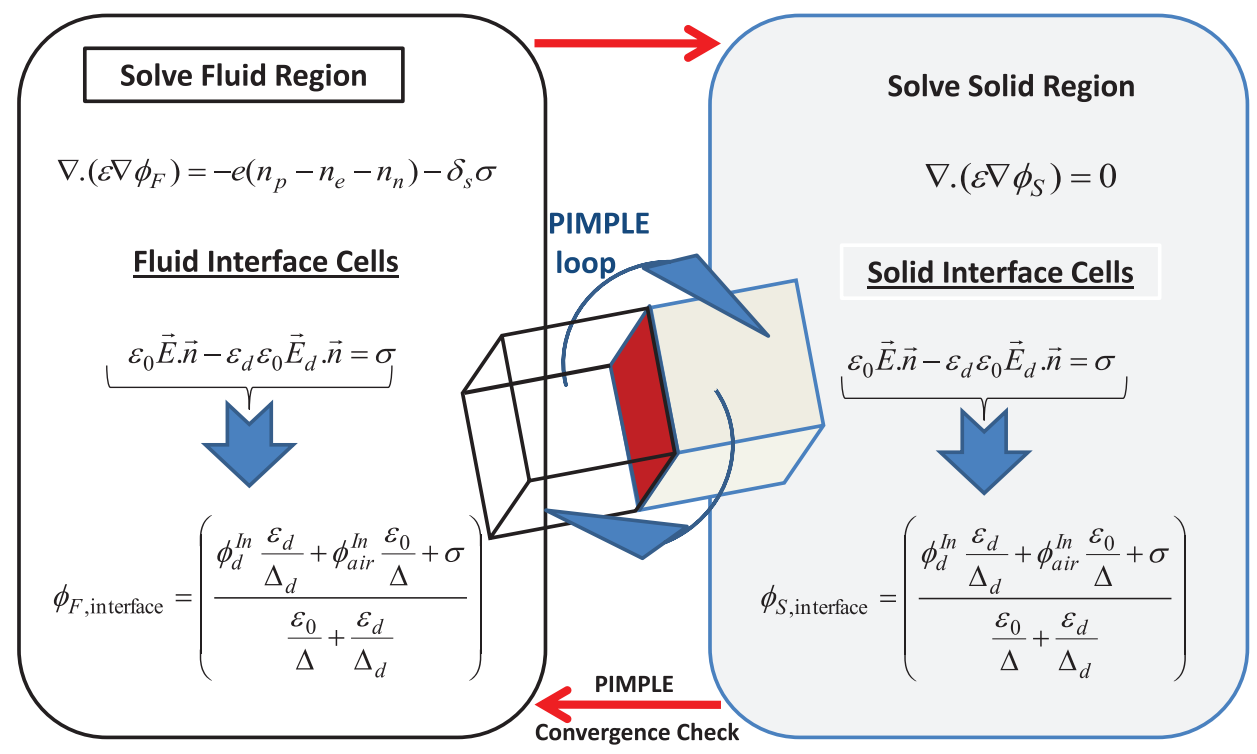

Fig. 2. Schematic of the partitioned approach for the plasma-fluid problem.

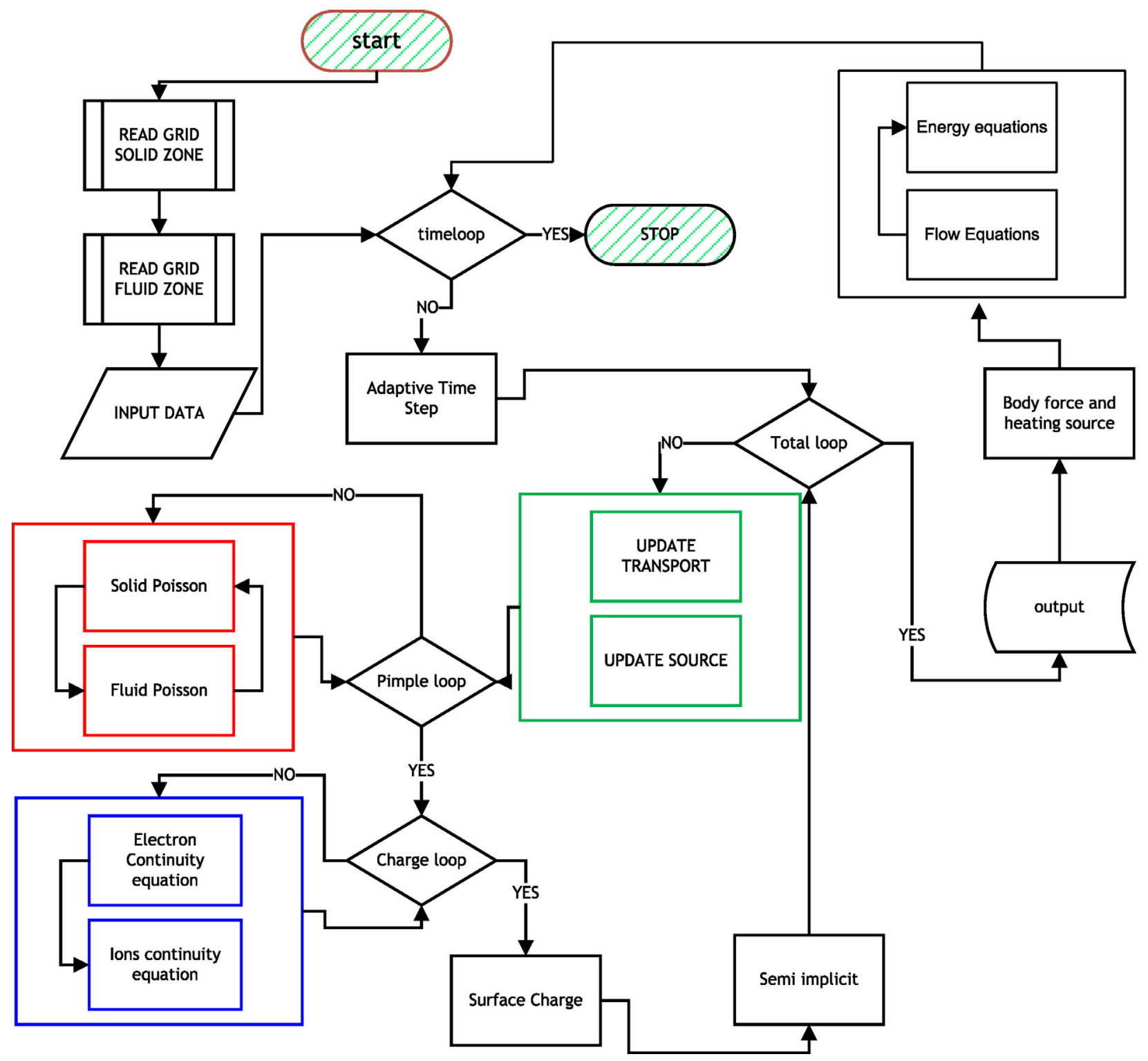

Fig. 3. Flowchart. 

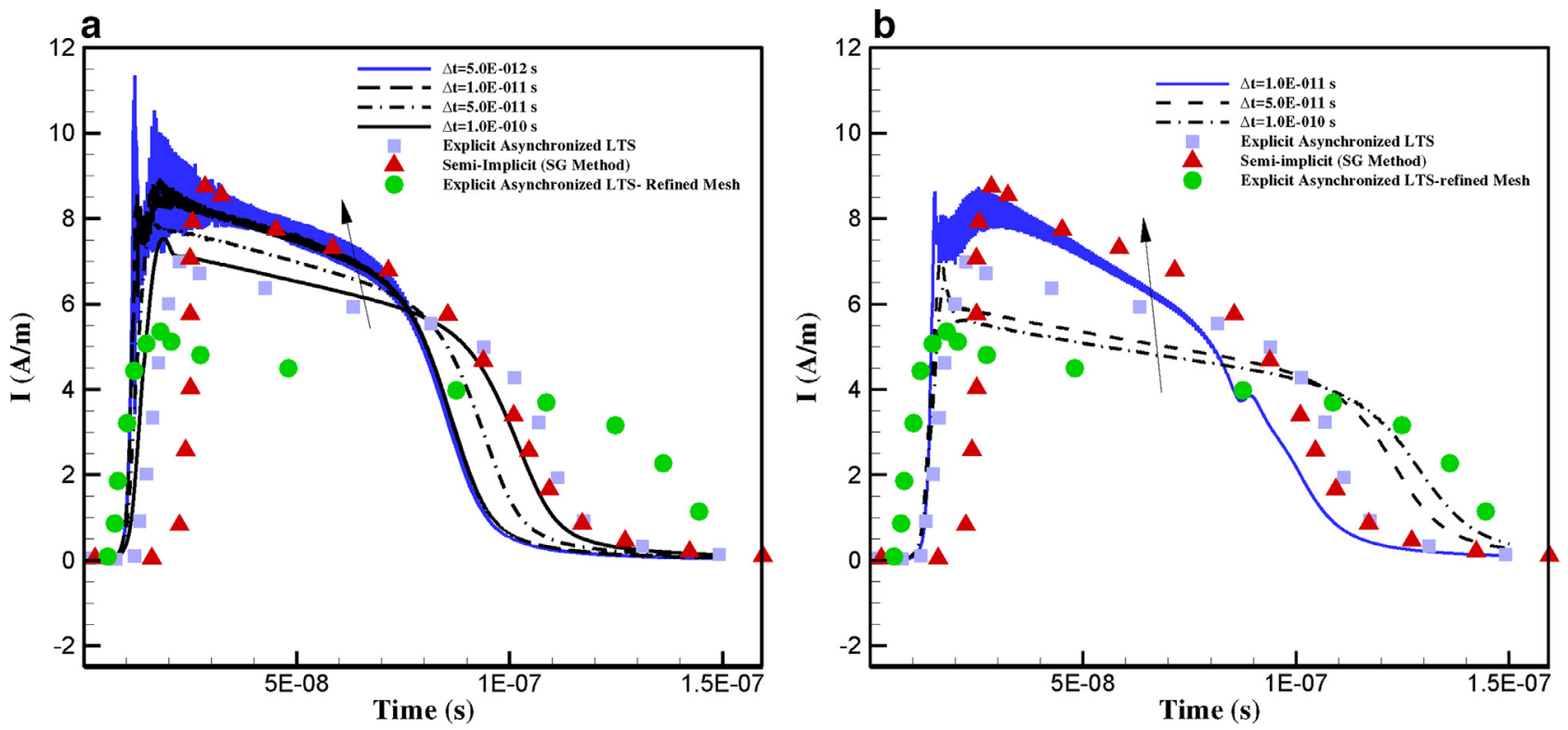

Fig. 4. Influence of time step of implicit Euler scheme on the current density: (a) first order upwind scheme; (b) second order deferred correction Gamma scheme.
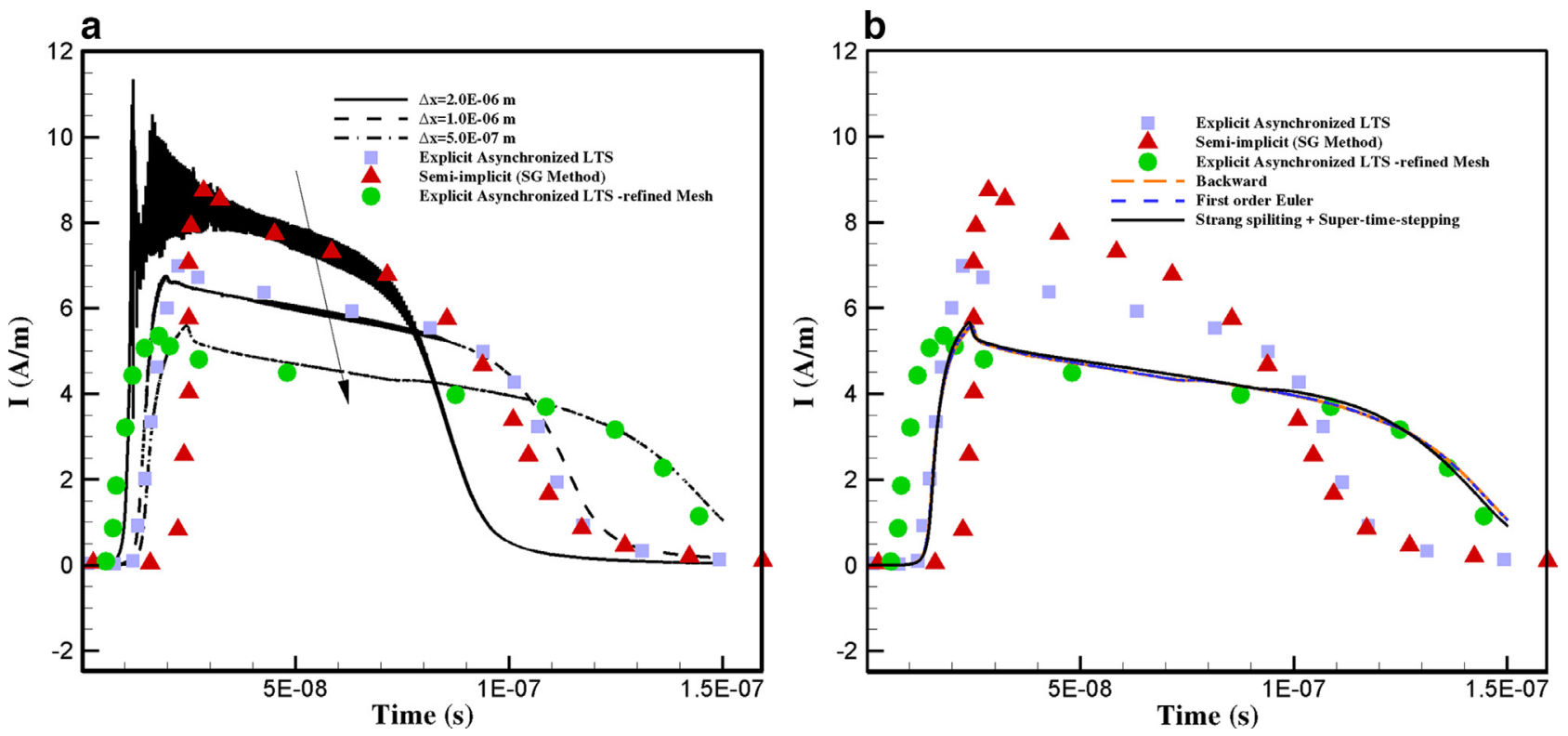

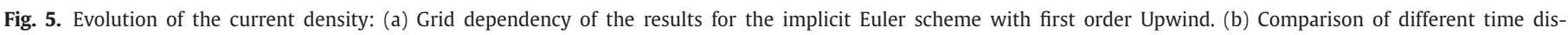
cretization for $\Delta t=5 \times 10^{-12} \mathrm{~s}, \Delta x=0.5 \mu \mathrm{m}$.

electric field and electron-ion density gradients relevant to the ionization wave front, and to ensure the plasma sheath near the wallgas interface can be adequately resolved, while balancing the consideration of the computational costs.

\section{Results and discussion}

Since there is no benchmark case for comparison, we have chosen the recent numerical work of Unfer [56] and Boeuf and Pitchford [21] (identified here respectively by Explicit Asynchronized LTS and Semi-Implicit (SG Method)) for the purpose of assessing of the influence of the each numerical issue previously described for alleviating the stiffness of the numerical solution of the plasmafluid model. Firstly, a simple case of constant positive voltage is considered. Although this case is simple, it can provide the possibility of correctly analyzing different numerical issues. This case comprises a 2D geometry with an exposed electrode as the anode (with constant positive voltage). Secondly, we will present some additional validation cases, on basically the same geometry but with different voltage waveforms.

One of the important aspects of the numerical simulation of plasma dynamics is the proper choice of a global time step $\Delta t$, a decision that requires an adequate balancing of computing efficiency with stability. In Fig. 4, the effect of the time step on the accuracy of the current density behavior is shown for a coarse grid with the cell length of $2 \mu \mathrm{m}$. The current represented is the displacement current on the bottom electrode. In Fig. 4a, the upwind scheme is used for the discretization of the convective term in the continuity equation and the Euler scheme is used for treating the unsteady terms. The results are clear in showing that when the time step is large, the solution is more diffusive and the peak value of the current happens later and has a smaller maximum magnitude. In addition, computation becomes unstable when the time step is reduced below $10^{-11} \mathrm{~s}$. In Fig. $4 \mathrm{~b}$, a second order 


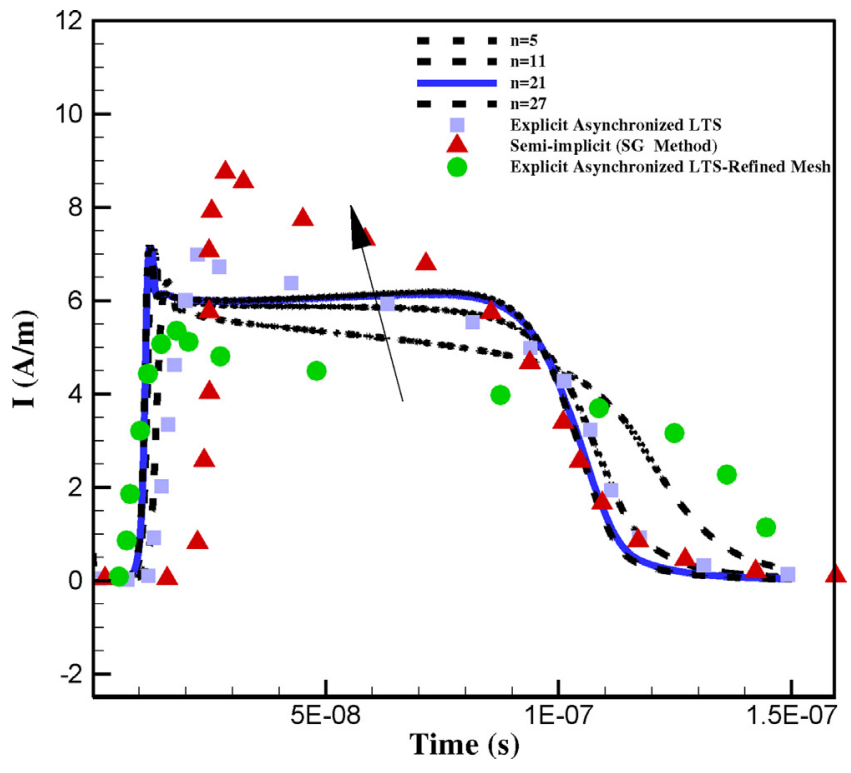

Fig. 6. Influence of the super-time-stepping levels on the resolution of the current density behavior for the case of positive constant voltage.

deferred-correction gamma scheme is applied for the convective terms. Comparing Fig. 4a and b, we see that the upwind scheme, as expected, shows a more diffusive behavior in comparison with the second order gamma scheme. While this kind of diffusive error makes the scheme highly stable even for large time steps, it produces wrong physical solutions.

In Fig. 5a, the grid independency test results are presented for simulations with Euler discretization in time and first order upwind spatial discretization. When the grid density is low, the effect of numerical diffusion appears to me more intense, and in contrast when the grid is refined, the resolution of the results is better. Moreover, as shown in Fig. 5b, all the time discretization schemes including the Euler, backward and splitted super-timestepping present similar accuracy on the refined grid with sufficiently small time steps.

We have also investigated the super-time-stepping which allows the schemes to overcome the CFL condition. In Fig. 6, the effect of the number of the super-time-stepping stages with RKL is demonstrated on a medium refined grid with $\Delta x=1 \mu \mathrm{m}$ and $\Delta t=5 \times 10^{-12} \mathrm{~s}$. For lower number of super-time-stepping stages, the results are more diffusive. This was expected, as an increase of $n$ should allow larger time steps to be used for the simulation. However, increasing further the number of the super-timestepping, in this case more than 21, did not change the resolution of the results obtained. We should mention that, while splitted super-time-stepping is an important technique for improving accuracy and stability, its ability to increase the global time step is limited. Although the results in Fig. 6 show that super-time-stepping allows much larger time steps than the limitation imposed by the CFL condition of explicit schemes, they do not prove that the savings in computer time are as substantial as with implicit schemes.

Up to now the influences of the main numerical treatments were shown for a positive constant voltage case, for which we can readily select the numerical approach for the simulations. In the following we will present validation studies for different voltage waveforms. In Fig. 7, the current density behavior in the case of a constant negative voltage and positive nano-second voltage is shown and the results are compared with numerical results of Boeuf and Pitchford [21] and Unfer and Boeuf [57]. The results of the present study are correctly capturing the current evolution versus time.

In Fig. 8, the current density behavior is analysed for the cases of a positive and a negative voltage ramp. The results show similar features as described in previous papers $[16,17,19]$ and are qualitatively valid when compared with those of these authors. It is clear from the results that there is a difference between the discharge characteristic in these cases. In the case of positive ramp, the electrode above the dielectric layer plays the role of an anode. Once the size and density of the positive ion cloud reach critical values, a high current breakdown occurs, characterized by the development of high amplitude pulses in the current profile. The discharge during the positive part of the cycle is composed of successive phases of ion cloud formation and high current breakdown. When a negative voltage ramp is applied, the current profile is also composed of current pulses, but with a frequency much larger than in the positive ramp case and of much lower intensity.

In the last case, an $\mathrm{AC}$ sinusoidal voltage with frequency of $8 \mathrm{kHz}$ and amplitude of $8 \mathrm{kV}$ is applied to the exposed electrode of the DBD and the results are shown in Fig. 9. These results are again a

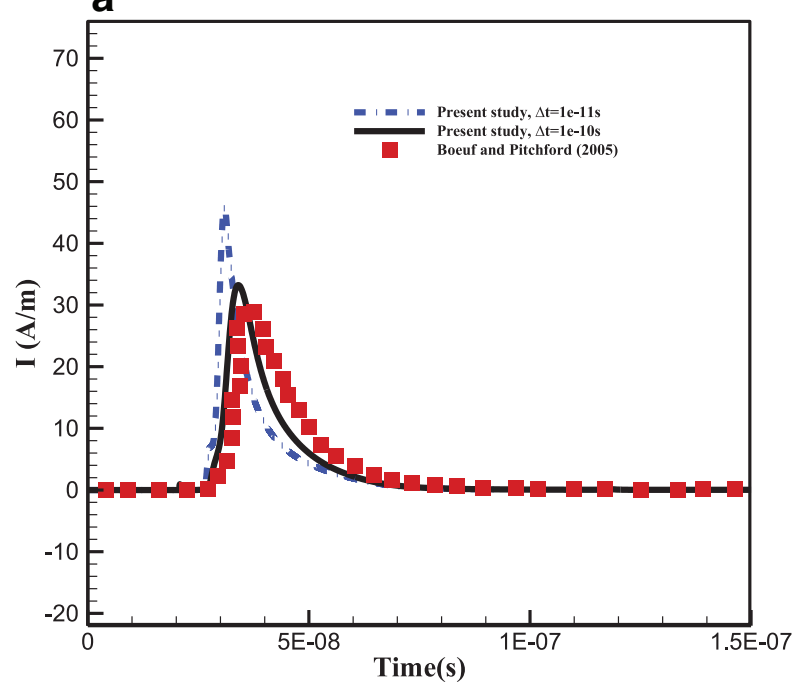

b

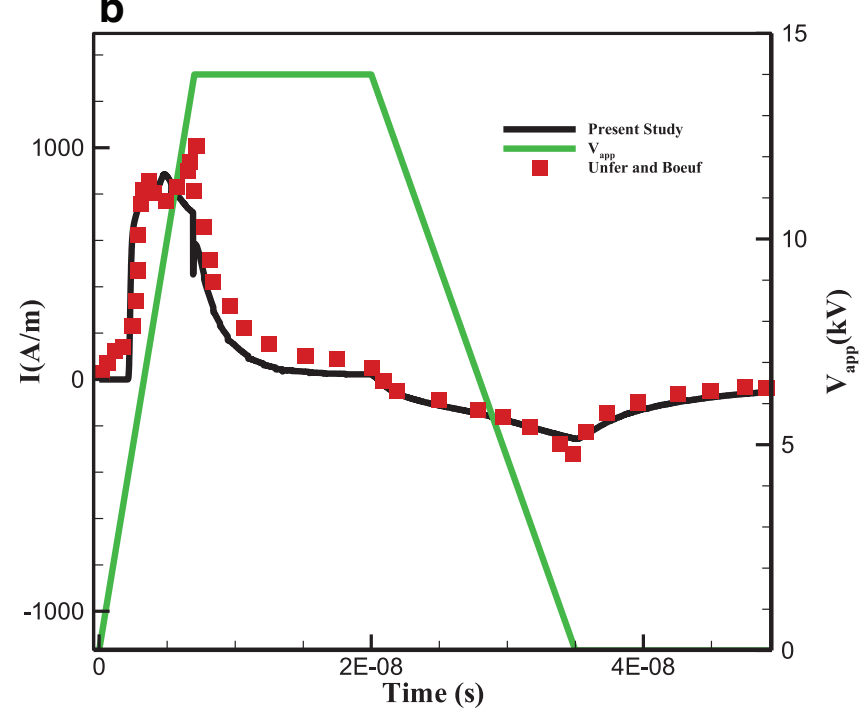

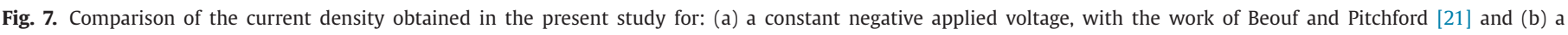
nano-second pulsed voltage, with the work of Unfer and Boeuf [57]. 

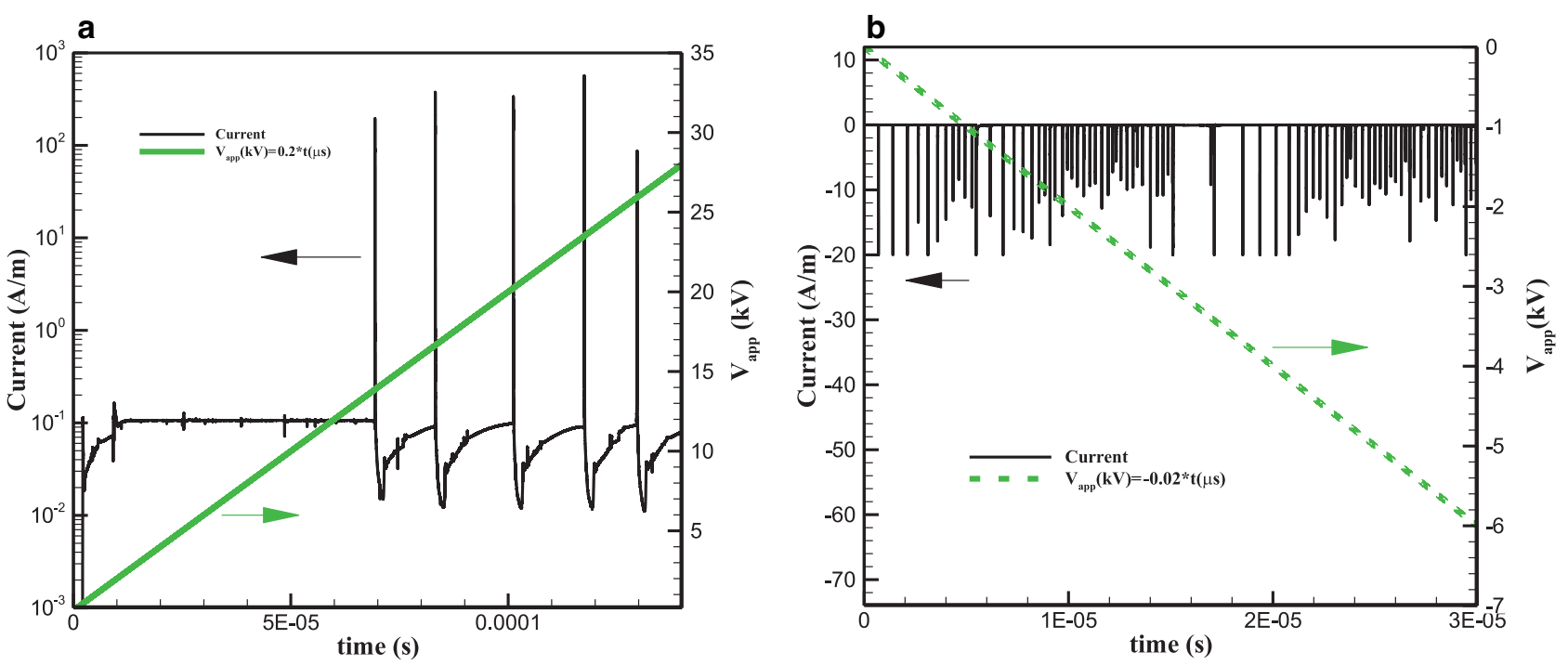

Fig. 8. Current characteristic of the dielectric barrier discharge with: (a) positive ramp voltage and (b) negative ramp voltage.

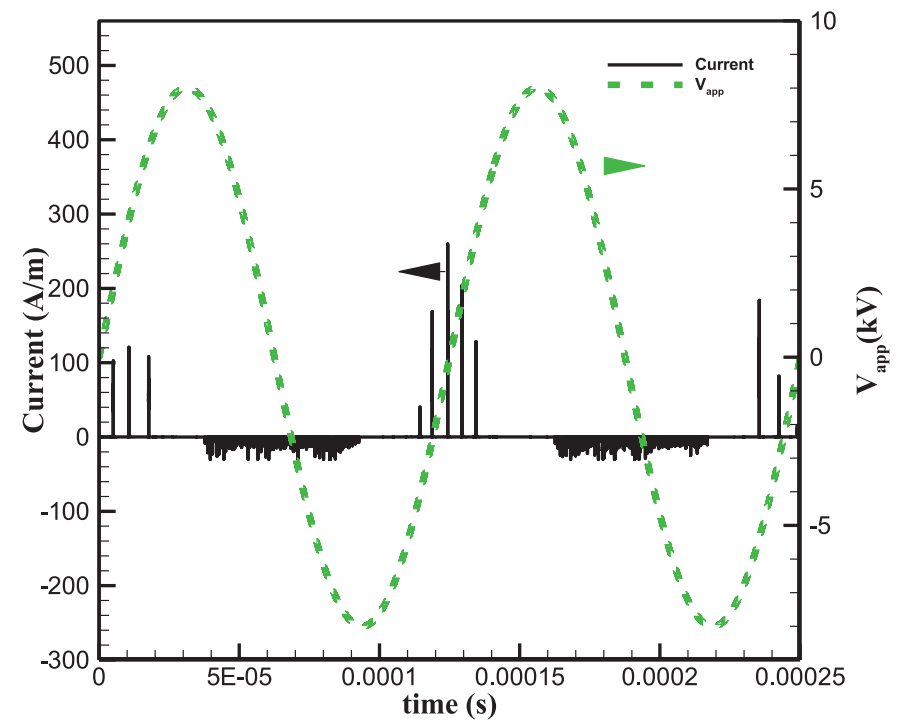

Fig. 9. Voltage and current of a DBD actuator with an AC-sinusoidal applied voltage.

in qualitative agreement with results presented in [17,58]. The differences observed in Fig 8 between the positive and negative ramp voltages are replicated in Fig. 9 for the ascending and descending parts of the AC sinusoidal voltage. This difference implies that the EHD force is important not only during the positive part of the sinusoidal voltage cycle but also during the negative part of the cycle.

The component of the force parallel to the plate is shown in Fig. 10 for a duration of the voltage similar to Fig. 9. During the positive part of the cycle, the EHD force is due to the formation of a positive ion cloud which is periodically interrupted by high current breakdown. The EHD force during the negative part of the cycle is due to the development of a negative ion cloud that continuously grows during the successive high frequency current pulses which form in this regime [19].

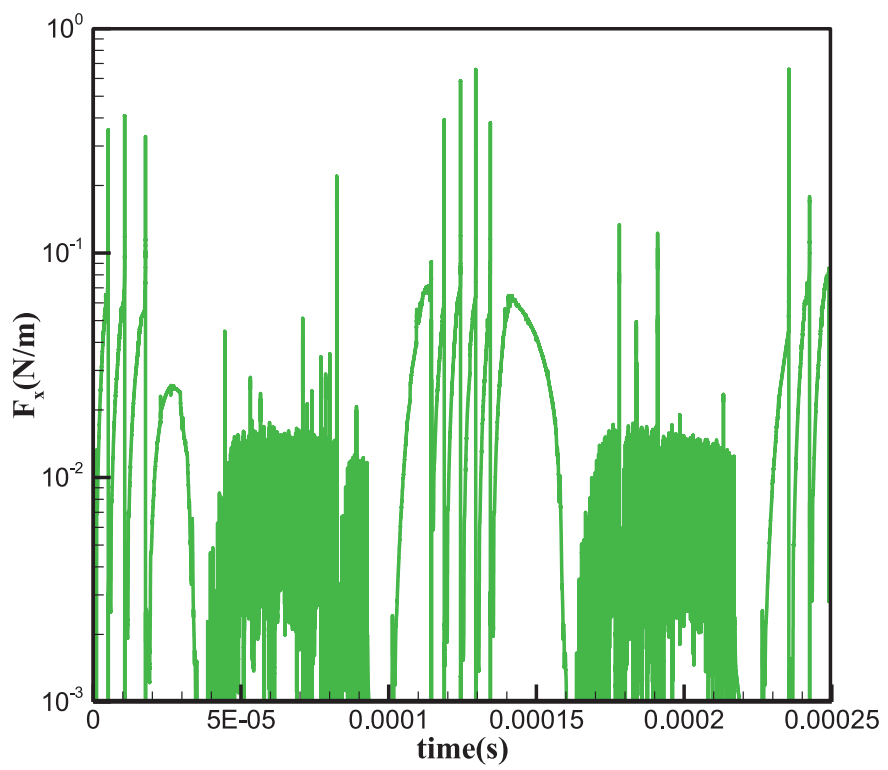

Fig. 10. Component of the EHD force parallel to the surface for actuation with AC sinusoidal voltage.

\section{Conclusions}

Different assumptions and considerations regarding the plasma fluid model are discussed. In addition, various numerical issues regarding the stability and accuracy of the methods are also discussed and explained. To solve these issues, a number of numerical remedies proposed in literature were implemented and tested. To this aim, a three-particle fluid model of plasma discharge in air, which is able to provide the spatial distribution and the time evolution of the charged particle densities, electric field and surface charges, was implemented in OpenFOAM. Different voltage waveforms, including positive and negative constant and ramp voltages, and a case of AC sinusoidal voltage have been considered for testing the accuracy of the implementation. 


\section{Acknowledgments}

The research work was supported by FCT Project PTDC/EMSENE/5742/2014 UNDULANT-Next (Unsteady boundary Layer flow control using plasma actuators of Next generation), part of the work was also supported by C-MAST (Center for Mechanical and Aerospace Science and Technology) FCT research center No. 151.

\section{References}

[1] Singh KP, Roy S. Vortical flow control on a conical fore body cross section using an array of pulsed dc actuators. J Appl Phys 2007;101:093301. doi:10.1063/1. 2720256.

[2] Likhanskii AV, Shneider MN, Macheret SO, Miles RB. Modeling of dielectric barrier discharge plasma actuators driven by repetitive nanosecond pulses. Phys Plasmas 2007;14:073501. doi:10.1063/1.2744227.

[3] Abdollahzadeh M, Páscoa JC, Oliveira PJ. Two-dimensional numerical modeling of interaction of micro-shock wave generated by nanosecond plasma actuators and transonic flow. J Comput Appl Math 2013;270:401-16. doi:10.1016/j.cam. 2013.12.030.

[4] Post ML. Plasma actuators for sepration control on staintionary and osciliatary airfoils [Ph.D. thesis]. University of Notre Dame; 2004

[5] Bai J, Sun J, Zhang Q, Wang D, simulation of RF hydrogen discharges in a transverse magnetic field PIC. Curr Appl Phys 2011;11:S140-4. doi:10.1016/j. cap.2011.04.023.

[6] Jayaraman B, Shyy W, Thakur S. Modeling of dielectric barrier discharge and resulting fluid dynamics. In: Proceedings of 44th AIAA aerospace sciences meeting and exhibit; 2006. p. 1-20. doi:10.2514/6.2006-686.

[7] Abdollahzadeh M, Pascoa JC, Oliveira PJ. Modified split-potential model for modeling the effect of DBD plasma actuators in high altitude flow control. Curr Appl Phys 2014;14:1160-70. http://dx.doi.org/10.1016/j.cap.2014.05.016.

[8] Abdollahzadeh M, Pascoa JC, Oliveira PJ. Numerical design and analysis of a multi-DBD actuator configuration for the experimental testing of ACHEON nozzle model. Aerosp Sci Technol 2015;41:259-73. http://dx.doi.org/10.1016/j.ast. 2014.12.012.

[9] van Dijk J, Kroesen GMW, Bogaerts A. Plasma modelling and numerical simulation. J Phys D Appl Phys 2009;42:190301. doi:10.1088/0022-3727/42/19/ 190301.

[10] Jayaraman B, Shyy W. Modeling of dielectric barrier discharge-induced fluid dynamics and heat transfer. Prog Aerosp Sci 2008;44:139-91. doi:10.1016/j. paerosci.2007.10.004

[11] Georghiou GE, Papadakis AP, Morrow R, Metaxas AC. Numerical modelling of atmospheric pressure gas discharges leading to plasma production. J Phys D Appl Phys 2005;38:R303-28. doi:10.1088/0022-3727/38/20/R01.

[12] Jayramann B. Computational modeling of glow discharge-induced fluid dynamics [Ph.D. thesis]. University of Florida; 2006.

[13] Roy S. Flow actuation using radio frequency in partially ionized collisional plasmas. Appl Phys Lett 2005;86:101502. doi:10.1063/1.1879097.

[14] Singh KP, Roy S. Simulation of an asymmetric single dielectric barrier plasma actuator. J Appl Phys 2005;98:083303. doi:10.1063/1.2103415.

[15] Duan X, He F, Ouyang J. Uniformity of a dielectric barrier glow discharge: experiments and two-dimensional modeling. Plasma Sources Sci Technol 2012;21:015008. doi:10.1088/0963-0252/21/1/015008.

[16] Nishida H, Abe T. Numerical analysis of plasma evolution on dielectric barrier discharge plasma actuator. J Appl Phys 2011;110:013302. doi:10.1063/1. 3603001.

[17] Boeuf JP, Lagmich Y, Unfer T, Callegari T, Pitchford LC. Electrohydrodynamic force in dielectric barrier discharge plasma actuators. J Phys D Appl Phys 2007:40:652-62. doi:10.1088/0022-3727/40/3/S03.

[18] Unfer T, Boeuf J-P. Modeling and comparison of sinusoidal and nanosecond pulsed surface dielectric barrier discharges for flow control. Plasma Phys Control Fusion 2010;52:124019. doi:10.1088/0741-3335/52/12/124019.

[19] Lagmich Y, Callegari T, Pitchford LC, Boeuf JP. Model description of surface dielectric barrier discharges for flow control. J Phys D Appl Phys 2008;41:095205. doi:10.1088/0022-3727/41/9/095205.

[20] Roy S, Gaitonde DV. Force interaction of high pressure glow discharge with fluid flow for active separation control. Phys Plasmas 2006;13:023503. doi:10. 1063/1.2168404.

[21] Boeuf JP, Pitchford LC. Electrohydrodynamic force and aerodynamic flow acceleration in surface dielectric barrier discharge. J Appl Phys 2005;97:103307. doi:10.1063/1.1901841.

[22] Shang JS, Huang PG. Modeling of ac dielectric barrier discharge. J Appl Phys 2010;107:113302. doi:10.1063/1.3415526.

[23] Shang JS, Huang PG. Surface plasma actuators modeling for flow control. Prog Aerosp Sci 2014;67:29-50. doi:10.1016/j.paerosci.2014.01.001.

[24] Singh KP, Roy S. Modeling plasma actuators with air chemistry for effective flow control. J Appl Phys 2007;101:123308. doi:10.1063/1.2749467.

[25] Likhanskii AV, Shneider MN, Macheret SO, Miles RB. Modeling of dielectric barrier discharge plasma actuator in air. J Appl Phys 2008;103:053305. doi:10.1063/1.2837890.

[26] Unfer T, Boeuf J, Rogier F, Thivet F. Modeling of dielectric barrier discharge and coupling with computational fluid dynamics. In: Proceedings of 46th AIAA aerospace sciences meeting and exhibit; 2008. http://dx.doi.org/10.2514/ 6.2008-1375.
[27] Wang C-C, Roy S. Energy and force prediction for a nanosecond pulsed dielectric barrier discharge actuator. J Appl Phys 2012;111:103302. doi:10.1063/1. 4722202.

[28] Weller HG, Tabor G, Jasak H, Fureby C. A tensorial approach to computational continuum mechanics using object-oriented techniques. Comput Phys 1998;12:620. doi:10.1063/1.168744

[29] Kee RJ, Miller JA. A split-operator, finite-difference solution for axisymmetric laminar-jet diffusion flames. AIAA J 1978;16:169-76.

[30] Maestri M, Cuoci A. Coupling CFD with detailed microkinetic modeling in heterogeneous catalysis. Chem Eng Sci 2013;96:106-17.

[31] Asada T, Nonomura K, Aono H, Sato M, Okada K, Fujii K. LES of transient flows controlled by DBD plasma actuator over a stalled airfoil. Int J Comut Fluid Dyn 2015;29:215-29. http://dx.doi.org/10.1080/10618562.2015.1032271.

[32] Corke TC, Enloe CL, Wilkinson SP. Dielectric barrier discharge plasma actuators for flow control. Annu Rev Fluid Mech 2010;45:505-29. doi:10.1146 annurev-fluid-121108-145550.

33] Valerioti JA, Corke TC. Pressure dependence of dielectric barrier discharge plasma flow actuators. AIAA J 2012;50:1490-502. doi:10.2514/1.J051194.

[34] Scheubert P. Modelling and diagnostics of low pressure plasma discharges [Ph.D. thesis]. Technischen Universitat Munchen; 2001.

[35] Marchand KG. Computational model of one-dimensional dielectric barrier discharges [M.Sc. thesis]. Air force institude of technology; 2005.

[36] Charles CS. Computaional modeling of the dielectric barrier discharge (DBD) device for aeronautical applications [M.Sc. thesis]. Air Force Institute of Technology; 2006

[37] D. Marshall, A. Federov, V. Soloviev, Mathematical fluid dynamics of plasma control over high speed wings, Reposit ADA536200, 2009.

[38] Sitaraman H, Raja LL. Simulation studies of RF excited micro-cavity discharges for micro-propulsion applications. J Phys D Appl Phys 2012;45:185201. doi:10. 1088/0022-3727/45/18/185201

[39] Jayaraman B, Cho Y-C, Shyy W. Modeling of dielectric barrier discharge plasma actuator. J Appl Phys 2008;103:053304. doi:10.1063/1.2841450.

[40] Fiala A, Pitchford L, Boeuf JP. Two-dimensional, hybrid model of lowpressure glow discharges. Phys Rev E 1994;49:5607-24. http://dx.doi.org/10 1103/PhysRevE.49.5607.

[41] Wang C, Roy S. Numerical simulation of a gas turbine combustor using nanosecond pulsed actuators. In: Proceedings of AIAA aerospace sciences meeting and exhibit; 2013. p. 1-17.

[42] Martens T, Bogaerts A, Brok W, van Dijk J. Computer simulations of a dielectric barrier discharge used for analytical spectrometry. Anal Bioanal Chem 2007;388:1583-94. doi:10.1007/s00216-007-1269-0.

[43] Morgan W, Boeuf JP, Pitchford LC. BOLSIG Boltzmann solver monument. USA: Toulouse France; 1996

[44] Jayaraman B, Thakur S, Shyy W. Modeling of fluid dynamics and heat transfer induced by dielectric barrier plasma actuator. J Heat Transf 2007;129:517. doi:10.1115/1.2709659.

[45] Balcon N. Atmospheric pressure radio frequency discharges, diagnostic and numerical modeling [Ph.D.thesis]. Australian National University; 2007.

[46] Hagelaar GJM. Modeling of microdischarges for display technology [Ph.D. thesis]. Technische Universiteit Eindhoven; 2000.

[47] Hagelaar GJM, De Hoog FJ, Kroesen GMW. Boundary conditions in fluid models of gas discharges. Phys Rev E 2000;62:1452-4.

[48] Arpa R, D'Ambrosio D. High-order accurate implicit scheme for drift-diffusion equations and application to dielectric barrier discharges. In: Proceedings of the 40th AIAA plasmadynamics lasers conference; 2009. p. 1-17. doi:10.2514/ 6.2009-3909.

[49] Strang G. On the construction and comparison of difference schemes. SIAM J Numer Anal 2013;5:506-17, http://dx.doi.org/10.1137/0705041.

[50] Tyson R, Randall LGS. Fractional step methods applied to a chemotaxis model. Math Biol 2000;475:455-75, http://dx.doi.org/10.1007/s002850000038.

[51] Ropp DL, Shadid JN, Ober CC. Studies of the accuracy of time integration methods for reaction-diffusion equations. J Comput Phys 2004;194:544-74. doi:10.1016/j.jcp.2003.08.033.

[52] Likhanskii A. Study of plasma phenomena at high electric fields in applications for active flow control and ultra-short pulse laser drilling [Ph.D. thesis]. The Pennsylvania State University; 2009.

[53] Meyer CD, Balsara DS, Aslam TD. A stabilized Runge-Kutta-Legendre method for explicit super-time-stepping of parabolic and mixed equations. J Comput Phys 2014;257:594-626. doi:10.1016/j.jcp.2013.08.021.

[54] Meyer CD, Balsara DS, Aslam TD. A second-order accurate super timestepping formulation for anisotropic thermal conduction. Mon Not R Astron Soc 2012;422:2102-15. doi:10.1111/j.1365-2966.2012.20744.X.

[55] Hagelaar GJM, Kroesen GMW. Speeding up fluid models for gas discharges by implicit treatment of the electron energy source term. J Comput Phys 2000;159:1-12. doi:10.1006/jcph.2000.6445.

[56] Unfer T. Méthode asynchrone pour la modélisation d'actuateurs plasma destinés au contrôle d'écoulement [Ph.D. thesis]. UNIVERSITÉ DE TOULOUSE; 2008.

[57] Unfer T, Boeuf JP. Modelling of a nanosecond surface discharge actuator. J Phys D Appl Phys 2009;42:194017. doi:10.1088/0022-3727/42/19/194017.

[58] Boeuf JP, Lagmich Y, Pitchford LC. Contribution of positive and negative ions to the electrohydrodynamic force in a dielectric barrier discharge plasma actuator operating in air. J Appl Phys 2009;106:023115. 